\title{
DR. 1266
}

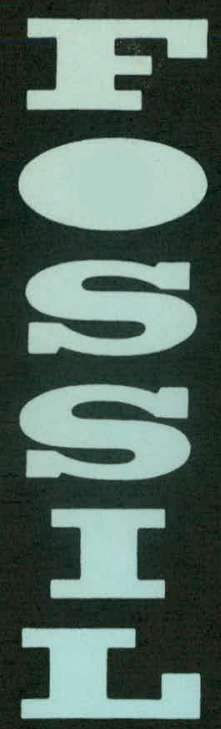

EVALUATION OF AN AUTOMATED REFLECTANCE

MICROSCOPE SYSTEM FOR COAL CHARACTERIZATION

Technical Report 18

By

D. S. Liscinsky

F. Vastola

January 1980

Date Published

Work Performed Under Contract No. EX-76-C-01-2030

The Pennsylvania State University

University Park, Pennsylvania

\section{U. S. DEPARTMENT OF ENERGY}




\section{DISCLAIMER}

This report was prepared as an account of work sponsored by an agency of the United States Government. Neither the United States Government nor any agency Thereof, nor any of their employees, makes any warranty, express or implied, or assumes any legal liability or responsibility for the accuracy, completeness, or usefulness of any information, apparatus, product, or process disclosed, or represents that its use would not infringe privately owned rights. Reference herein to any specific commercial product, process, or service by trade name, trademark, manufacturer, or otherwise does not necessarily constitute or imply its endorsement, recommendation, or favoring by the United States Government or any agency thereof. The views and opinions of authors expressed herein do not necessarily state or reflect those of the United States Government or any agency thereof. 


\section{DISCLAIMER}

Portions of this document may be illegible in electronic image products. Images are produced from the best available original document. 


\section{DISCLAIMER}

"This book was prepared as an account of work sponsored by an agency of the United States fovernment. Neither the United States Government nor any agency thereuf, nur any of their employees, makes any warranty, express or implied, or assumes any legal liability or responsibility for the accuracy, completeness, or usefulness of any information, apparatus, product, or process disclosed, or represents that its use would not infringe privately owned rights. Reference herein to any specific commercial product, process, or service by trade name, trademark, manufacturer, or otherwise, does not necessarily constitute or imply its endorsement, recommendation, or favoring by the United States Government or any agency thereof. The views and opinions of authors expressed herein do not necessarily state or reflect those of the United States Government or any agency thereof."

This report has been reproduced directly from the best available copy.

Available from the National Technical Information Service, U. S. Department of Commerce, Springfield, Virginia 22161.

Price: Paper Copy $\$ 7.00$

Microfiche $\$ 3.50$ 


\section{MASTER}

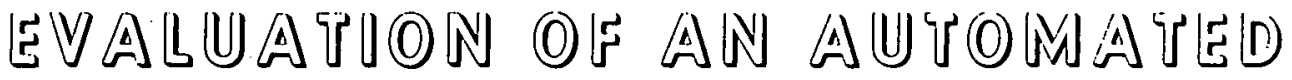

RBELESTANCB MUCROSCOPBS SYSTEM

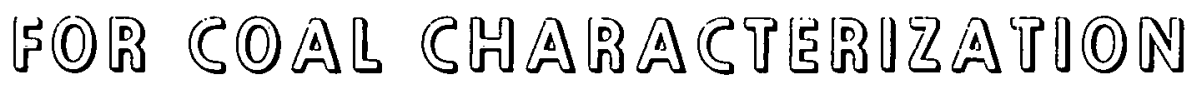

\section{Technical Report 18}

Prepared by

D.S. Liscinsky and F. Vastola

at

THE PENNSYLVANIA STATE UNIVERSITY

PSU-TR-18



Date Published - January 1980

PREPARED FOR

UNITED STATES DEPARTMENT OF ENERGY

Under Contract No. EX-76-C-01-2030 







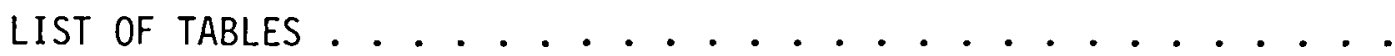

LIST OF FIGURES ..............................

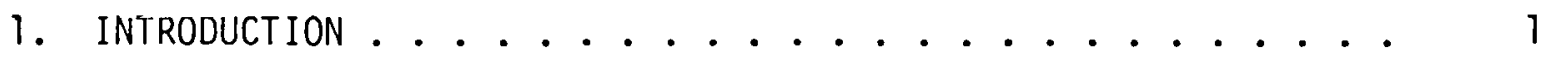

1.1 Statement of the Problem ............ 3



2.1 Properties of Macerals ............ 5

2.2 Identification of Macerals ........... 6

2.3 Measurement of Reflectance ........... . 7

2.4 Automated Reflectance Microscope Techniques ...... 8

3. EXPERIMENTAL APPARATUS AND PROCEDURE .......... 12

3.1 Automated Reflectance Microscope Concept ....... 12

3.2 Automated Reflectance Microscope Apparatus . . . . . . . 12

3.3 Data Acquisition Software ............... 14

3.4. Experimental Procedure ............. 16

3.5 Treatment of the Data ............ 19

3.6 Verification of the Data Acquisition System ...... 21

4. RESULTS AND DISCUSSION ..................... 26

4.1 Interpretation of the Reflectogram ........ 26

4.2 Improvement of the Reflectogram .......... 28

4.3 Data Processing Techniques ........... 30

4.4 Data Analysis Using a Connectivity Algorithm ..... . 31

4.5 Bimodal Samples..................... 41



5.1 Summary ...................... 57

5.2 Suggestions for Future Research ................. 59



APPENDIX A: Organization of the Data-Acquisition Unit Built for the Automated Reflectancc Microscope Systemi . . 65

APPENDIX B: Determination of a Particle Size Distribution from a Curve Representing a Collection of Spheres of Two Different Diameters.......... 66

APPENDIX C: Description of the Connectivity Algorithm . . . . 67 


\section{LIST OF TABLES}

$\underline{\text { Table }}$

Page

1 Composition of Synthetic Pellets Used to Examine the Connectivity Algorithm ............ 42

2 Calculation of the Expected Amount of Microstructure Using a Simple Spherical Model . . . . . . . 


\section{LIST OF FIGURES}

Figure

$\underline{\text { Page }}$

1 Schematic Diagram of the Automated Reflectance

Microscope System .............. 13

2 Data Stream of PSOC-328 . . . . . . . . . 20

3 A Reflectogram of PSOC-328 ........... 22

4 Reproducibility of a Reflectogram ......... 23

5 Reflectograms of PSOC-328 Acquired Simultaneously on a PDP-8 and KIM-1 Computer........... 25

6 A Series of Reflectograms on Plot Types I, II, III, and IV ...............

7 A Series of Reflectograms Representing Particle Sizes of Greater than or Equal to 2, 4, 8, and

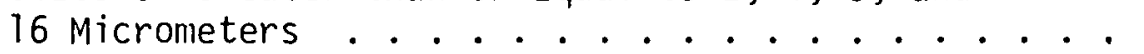

8 A Series of Reflectograms Representing Particle Sizes of Less than $4,8,16$, and 32 Micrometers . . . . 36

9 A Series of Reflectograms Showing the Percentage Change Between Particle Sizes of $>2$ Micrometers and Particle Sizes of $\geq 4, \geq 8, \geq 76, \geq 32$

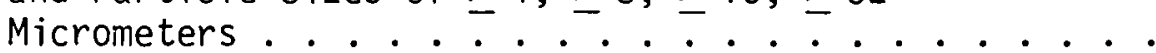

10 Data Stream of a Pellet with a Low Concentration of Coal ............... 40

11 Reflectogram of Pellet Number 1........ 44

12 A Series of Reflectograms for Coals of Increasing

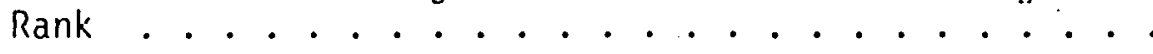

13 PSOC-102, PSOC-409, PSOC-102, PSOC-409 and Pel let Number 1..............

14 Reflectogramis of Pellets Number 2, 1, and 4.... 48

15 Reflectograms of Pellet Number 1 at Particle Sizes of Greater than or Equal to 2, 4, 8, and 16 Micrometers ................

16 Reflectograms of PSOC-013, $\mathrm{AO}_{\mathrm{e}-1}$ at Particle Sizes of Greater than or Equal to 2 and 4 Micrometers and Less than 4 Micrometers (Tnp to Bottom) ...... 


\section{LIST OF FIGURES (Continued)}

Figure

Page

17 Schematic Diagram of the Data-Acquisition System . . . 65

18 Flow Chart of the Connectivity Algorithm ...... 68 


\section{INTRODUCTION}

The complex nature of a coal results from the many different parent materials from which it originated. These materials consisted of a wide variety of plant forms and inorganic sediments. Together this mixture underwent a progressive series of alterations to form coal. Consequently coal is a physically and chemically heterogeneous substance.

For optimum coal utilization it is necessary to be able to relate the properties of a coal to its behavior in an industrial process. The establishment of such a relationship allows selection of the best $\operatorname{coal}(s)$ for a given process, or the modification of process conditions to suit the available coal(s). Therefore it is important to classify coals in a way that aids in utilization.

Many characteristics of coals are important in determining a coals behavior in industrial processes. Two of the more studied parameters are rank and petrographic composition. Rank refers to the degree of alteration, or metamorphism, a coal has undergone in its continuous evolution toward a total carbon structure. Petrographic composition is based on the ability to quantitatively identify the individual components in a coal. Both of these parameters are of practical industrial importance in defining the properties of coals.

Rank is ail important classification parameter since a large number of properties of a coal vary in a predictable manner with metamorphism. These include carbon content, volatile matter, reflectance, and many others. The industrial importance of rank is based on the similar properties pussessed by coals within certain 
ranges of rank. Therefore rank is often used to determine the suitability of a certain coal for a given process.

Most classification systems rely on simple laboratory tests such as carbon content, percent volatile matter, calorific value, or swelling to describe rank. These measurements can be misleading, since they represent the average of the heterogeneous character of a coal. Ideally more than one parameter should be used to establish rank, but practical considerations often limit testing.

Petrographic studies have led to the use of reflectance measurement as a more accurate index of coai rank. One particular microscopic constituent in coal, vitrinite; shows a marked change in reflectance with metamorphism. Reflectance measurements of vitrinite to establish rank correspond quite well with the more widely used carbon content and volatile matter tests. However, unlike chemical tests to determine rank, direct measurement of an individual coal component is less subject to changes in the overall petrographic composition of the coal. For this and other reasons many researchers judge reflectance to be the best single rank parameter (1).

In recent years it has become recognized that further characterization of a coal is often necessary. One of the techniques that is increasingly used is the quantitative description of a coal in terms of optically distinct homogeneous species called macerals. This is referred to as petrographic analysis. The analysis is based on the microscopic identification of macerals. These entities originate from associations of the various organic deposits that formed the coal. Therefore the properties exhibited by these individual 
components partly determine the overall properties of a coal. Simple chemical tests cannot account for this variability.

The close correlation of petrographic composition with the chemical and physical properties of a coal has been successfully used to predict coke strength and coke performance (2). Such predictions allow blending of different coals to obtain optimum coking properties. In addition to using petrographic analys is to predict coking behavior it is also probable that correlations will be found for gasification and liquefaction processes. Therefore determination of the petrographic composition is important as an accurate index of the potential use of a coal.

\subsection{STATEMENT OF THE PROBLEM}

Detailed characterization of a coal is time consuming and difficult due to the heterogeneous nature of coal. However efficient utilization requires an accurate and relatively rapid description of its properties. In this respect the determination of rank and petrographic composition are two of the more important parameters used to describe a coal.

Unfortunately petrographic analysis is such a time-consuming procedure that only a limited number of samples are analyzed. In addition the precision of the analysis is limited by the relatively sillall number of measurements that are made per samplc and the ability of the operator to accurately identify the various macerals. The need for a more rapid analysis has lead to the development of automated techniques. 
Automated systems have the potential advantages of being rapid, completely objective, and highly accurate as large populations of measurements can be accumulated. Also many types of analyses can be performed simultaneously, such as: petrographic composition, meanrandom vitrinite reflectance (rank), pyrite concentration, and particle size distribution.

Implementation of completely automated systems is not without problems. The intent of this study is to evaluate the effect of various parameters on the data obtained by a rapid-scan system. Better understanding of the system limitations, and what can be done about them, will allow demonstration of the characterization potential of automated reflectance microscope techniques. 


\section{BACKGROUND}

\subsection{PROPERTIES OF MACERALS}

Macerals can be split into two broad groups based upon their behavior during carbonization. The first group consists of reactive components and includes primarily vitrinites and exinites. The second group consists of inert components collectively referred to as inertinites.

By far the most important coal maceral is vitrinite. Besides being the most reactive component, it is found in all ranks of coal and rarely occurs in concentrations of less than $50 \%$ by volume in United States coals (overall mean is about 70\%). The behavior of vitrinites is characterized by plasticity and swelling during carbonization, thus giving a coal its agglomeration properties. Most reflectance measurements are made on vitrinite because of its high concentration, large particle size (less friable than other macerals), and homogeneous appearance.

Another important group of reactives is the exinites. These macerals are hydrogen-rich and less aromatic than the corresponding vitrinites. Upon carbonization they yield a high percentage of volatile matter and become even more fluid than vitrinites. The exinites are characterized by very distinct shapes, such as: long narrow bands, nvals, and rod=like filaments. The hydrogen rich nature of this group allows them to fluoresce when stimulated by ultra-violet radiation, and this fact is used for identification purposes. 
Inertinites of course remain inert upon carbonization. They serve as an aggregate material thereby adding strength to the coke product. These macerals are highty aromatic (greater than $90 \%$ carbon) and thus produce very little volatile matter upon heating.

\subsection{IDENTIFICATION OF MACERALS}

Neither the exact chemical composition or the structure of macerals is known. They are however chemically different as is evidenced by their behavior upon heating and physical separation, that is, reactivity and hardness. This can be attributed to the degree of aromaticity and ring condensation they possess (3).

As the degree of aromaticity (atomic density) of a structure increases, so does its refractive index. Ring condensation governs the mobility of electrons and thus the absorption of incident radiation. Both of these properties are indicative of the structure of a material and related to the reflectance of an absurbirily solid under vertically incident light by Beer's equaliun, which is:

$$
R=\frac{\left(n-n_{0}\right)^{2}+(n k)^{2}}{\left(n+n_{n}\right)^{2}+(n k)^{2}}
$$

where $n$ and $k$ are the refractive and absorptive indices of the solid and $n_{0}$ is the refractive index of the medium in which the measurements are made.

Therefore since macerals differ in chemical structure, reflectance can be used as an index of rank, since aromaticity and ring condensation tend to increase with metamorphism.

Although reflectance can uniquely identify a maceral, the natural size and shape in which they are found make recognition 
tedious using only reflectivity. Many macerals are small (less than 2 micrometers in diameter) making it difficult to get an accurate measurement. In addition, the anisotropic nature of coal means that the random orientation of macerals expected in a sample will result in a range of reflectance readings for any given maceral. Using a rotating stage and a polarized light source to record the maximum reflectance will reduce this problem, but makes for a time consuming procedure. Thus petrographic analysis is usually done by visual observation of morphology with a reflectance microscope (ASTM D 2799). With respect to the reflectance of macerals little has been published except on vitrinites, partly because it is the most suitable maceral for measurement. The relative reflectances of exinites, vitrinites, and inertinites are generally different at levels of rank less than $90 \%$ carbon (4). However, a potential problem in using reflectance to identify macerals is that each maceral group has many associated types due to different origins and formation conditions. Therefore any given maceral will have a reflectance distribution rather than a discrete value.

\subsection{MEASUREMENT OF REFLECTANCE}

Early techniques of measuring reflectance were aimed at studying the variation of vitrinite reflectivity with rank $(5,6,7)$. Controversy over this relationship raged for many years before being resolved by increased accuracy of modern equipment and larger data bases.

Many of the early determinations made use of the Berek microphotometer (8). Essentially this instrument is a microscone attachment 
which permits visual comparison of a reference beam and a beam reflected by the sample. The two are subsequently matched in intensity by rotation of a nicol prism. The change in intensity of the reference beam is proportional to the square of the sine of the angle of rotation of the prism. Thus a ratio can be set up between the unknown sample and a standard of known reflectance and the calculation made. A later improvement was made (9) by introducing neutral density filters to decrease the reference intensity and increase the variation between the beams. This increased the required angle of rotation and thus the accuracy of the measurement.

The Berek microphotometer technique was limited by the subjectivity introduced by the observer. Therefore a photocell was next incorporated to measure the intensity of the two beams. By using a galvanometer to balance the two signals an objective measurement could be made. Such a dual beam system provided very reliable data, however, the excellent stability of modern equipment permits present methods to employ a sinqle beam technique. Calibration is performed on a standard reflecting material. The specifications and procedure for the measurement of vitrinite mean-maximum reflectance are given in ASTM method D 2798.

\subsection{AUTOMATED REFLECTANCE MICROSCOPE TECHNIQUES}

Marly attempts have been made at quarilitative automated microscopy. Despite the difficulties in using reflectance to identify macerals, most automated techniques use this measurement as the distinguishing criteria. The first attempt at quantitative microscopic analysis applied to coal was made in 1952 with a flying-spot 
microscope (10). The technique was not truly automatic since manual recording was required. Although the results were encouraging, the primitive state of electronic technology severely limited future development at that time.

The first fully automatic system was the Automatic Microscope Electronic Data Accumulator (AMEDA) system described originally by Bomberger and Duel (11) and later modified by Bayer, et al (12). The purpose of the system was to measure pyrite size distribution for use in coke and steel production. The system was successfully applied to measurements of pore volumes in coke, rust spots on tin plates, and pyrite size distributions in coal.

The system consisted of a reflectance microscope to focus the light, a photomultiplier tube to measure the light reflected, and an $x-y$ stage drive to move the sample. Although this equipment configuration is very good, the recording end of the system was somewhat inflexible. It consisted of a maximum of ten counters which were assigned to specific reflectance ranges. These ranges had to be assigned values prior to the run. Therefore it was difficult to chose sufficiently narrow ranges and yet still correctly describe the sample. However, reproducibility for samples with components that greatly varied in reflectivity such as rust versus tin, was good and encouraged further work.

Beginning in 1969, McCartney and Ergun (13), working at the U.S. Bureau of Mines, developed a system (based on the AMEDA principle) that involved the recording of individual reflectance readings on magnetic tape. The tape was then processed by computer 
to obtain a pyrite size distribution and later maceral composition. A unique feature of their system was a spiral stage drive. It was used to eliminate some of the vibration and focussing problems of $x-y$ drives, but the primary improvement was the ability to process the data by computer.

The speed of operation of the equipment proved to be a limiting factor, as a complete analysis took about 3.5 hours. Correlation of pyrite content and size distribution with chemical and visual analysis was quite good. However, correlation of the processed data with visual maceral composition proved difficult.

The processing technique that was used assigned each readings to one of eight predetermined reflectance levels. This was similar to the AMEDA interpretation except more versatile in that the reflectance levels could be modified without another run. To get the calculations to agree with the visual maceral analysis usually required arbitrary adjustment of these levels.

Kojima. et al (14), working at Nippon Steel Corporation, have developed an automated microscope system that appears to give the best results of any attempt to date. Although based on the AMEDA principle, they employ a pulsed stage and an electrical integrator to increase the accuracy of each individual reading. A medium sized computer records and analyzes the data.

Their technique involves generation of a histogram of reflectance readings. This data was then reduced by statistical treatment. Gaussian distributions are fitted to obtain the matrix and vitrinite percentages. Once these areas have been determined, they are removed 
from the main distribution. The remaining distribution is then interpreted so that various areas are assigned to various maceral groups. This involves elimination of unrealistic readings by statistical treatment and interpretation of the irregularities that remain after the matrix and vitrinite components are eliminated. The results they report compare very well with the vitrinite concentrations obtained by standard petrographic analysis, but the percentages obtained for other macerals is less satisfactory.

Previous work at The Pennsylvania State University $(15,16,17$, 18) has also demonstrated the usefulness of an automated microscope system. The system consisted of a reflectance microscope fitted with a photomultiplier tube and attached to a motorized $x-y$ stage drive. This equipment was interfaced to a minicomputer for data acquisition, storage, analysis, and display. Hartmann (17) has applied the system to pyrite chord-size distributions and found good.correlation with visual analysis. Pyritic sulfur content obtained by chemical analysis and by the system was also in good agreement. Frompovicz (16) obtained data to correlate the mean-random reflectance to vitrinite to the mean-maximum vitrinite reflectance obtained by ASTM methods. He was also able to use curve-matching techniques on reflectogram profiles to differentiate between coals. This was useful for predicting the properties of an unknown coal by generating a reflectogram and searching for similar coals in a small data base. 


\section{EXPERIMENTAL APPARATUS AND PROCEDURE}

\subsection{AUTOMATED REFLECTANCE MICROSCOPE CONCEPT}

The Automated Reflectance Microscope (ARM) system is diagrammed in Figure 1. A reflectance distribution of the pellet surface is obtained by using a stationary high intensity light source and a moveable stage. By digitizing the output of the photomultiplier tube at regular intervals, a series of readings corresponding to the intensity of light reflected by the surface are received by the microcomputer for processing.

\subsection{AUTOMATED REFLECTANCE MICROSCOPE APPARATUS}

A Leitz Ortholux MPV-1 Reflectance Microscope with an oil immersion objective is used to focus a stabilized beam of light from a 100 watt tungsten halogen lamp onto the polished surface of a coal pellet. The light reflected normal to the surface passes through a 544 nanometer interference filter and is measured by an Emitronics 6094B photomultiplier tube, which converts the photon flux to an electrical signal. The signal is proportional to the intensity of light reflected off an area of known dimension on the pellet surface. The photomultiplier signal, after being converted from a current to a voltage and passing through an amplifier and a low pass filter, is digitized to a resolution of .008 volts by a Datel E8B2 dual slope analog-to-digital converter. Scanning of the pellet surface is accomplished by a modified Femco AMEDA stage drive, which mnves the stage at a constant rate in a continuous $x-y$ raster. 


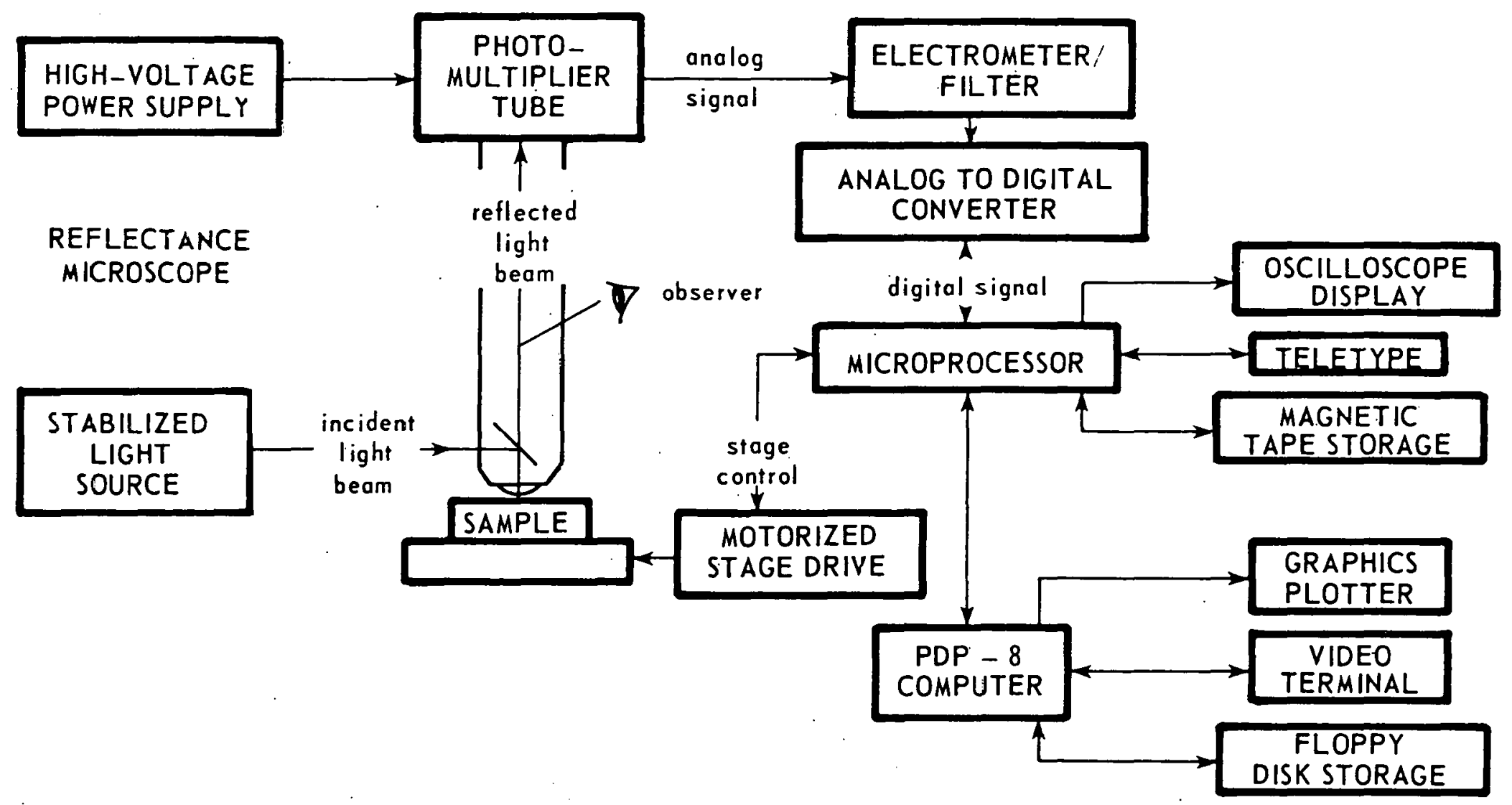

Figure 1. SCHEMATIC DIAGRAM OF THE AÜTOMATED REFLECTANCE MICROSCOPE (ARM) SYSTEM USED IN THIS STUDY 
The data acquisition section of the system was built around a 6502 microprocessor as configured in a KIM-1 microcomputer module by Conmodore Calculator Company. This unit is an eight-bit computer that has an operating system in read-only-memory and a choice of operating features. These include a keyboard and display included as part of the module, a teletype interface for hard-copy printing, and an audio cassette interface for magnetic tape storage and retrieval of programs and data. A more detailed description of the KIM-1 system can be found in the KIM-1 User Manual supplied by Commodore with the unit.

Although the microcomputer module was bought mounted on a Printed Circuit board, to make it operational required additional connections. In addition to a power supply and connections to use the teletype and cassette interfaces, the module was expanded to include additional random-access-memory for more elaborate programming and extra data storage. Also a 6530 peripheral interface adapter has been interfaced to provide external communication via two bidirectional ports. This device was used to operate two 8-bit digital-to-analog converters that are used to generate displays on an $x-y$ oscilloscope.

The micrucomputer data-acquisition unit was also interfaced to a ГOP-8. The dala can lhen be rurther processed or plotted on Houston Instrument Complot Plotter. A complete diagram of the dataacquisition system that was constructed is shown in Appendix A.

\subsection{DATA ACQUISITION SOFTWARE}

The actual operation of the data-acquisition system is performed by computer programs written on two levels. A program written in the 
programming language Basic issues prompts on a teletype that require the operator to enter responses to questions about run parameters. The program is semi-conversational in form, allowing almost anyone to operate the system with a minimum of instruction and little likelihood of error. Within this main program are subroutine calls to machine language programs. These programs perform the actual input and storage of data.

The package provides flexibility in choice of the total number of readings in a run and the data acquisition rate. Although the total number of readings that can be stored is quite large (17 million), each reflectance catagory is limited to a population of 65,536 . Therefore the practical limit for the total readings in a run is about 1 million, which is more than large enough.

The acquisition rate is limited by the conversion time of the analog-to-digital converter, which is 300 microseconds. Several microseconds are also required for program overhead. Therefore the maximum acquisition rate is about 3000 readings/second. Slower rates are typically used.

Several functional features are also provided. The keyboard display of the module is used to monitor the photomultiplier tube during standardization. During the run the oscilloscope is used to display the data array as it is being generated. This is a real-time display and is useful in spotting any gross miscalibrations at the beginning of a run. The same display can be used to observe and compare runs before time is taken to make a hard copy of the data. 


\subsection{EXPERIMENTAL PROCEDURE}

Before beginning a run, the equipment must be standardized. The potential applied to the photomultiplier tube is 920 volts. This voltage is below the upper saturation point of the tube, but high enough to maximize its signal-to-noise ratio. Using the electrometer gain, signals in the 0.0 to 2.0 volt range of the analog-to-digital converter can be selected. The response of the tube is linear in this range.

Standardization is accomplished by focussing the microscope on a Bausch and Lomb gilass standard with a reflectance of $1.006 \%$. The output voltage of the photomultiplier tube measured by the analogto-digital converter thus corresponds to $1 \%$ reflectance. This voltage can be adjusted with the electrometer gain. This adjustment determines the range of reflectance that is observable within the 0.0 to 2.0 volt operating range of the analog-to-digital converter. That is, setting the output to 0.5 volts means that the full scale reflectance value will be $4.0 \%$ ( 2 volts). The entịre reflectance range is used. which allows each reading to be measured to the nearest $0.016 \%$ reflectance.

Once standardization is complete, several other parameters must be set before the scan can begin. Several considerations determine their choice. The first consideration is the total population of readings in the run. It is important that this number be sufficiently large to obtain adequate statistics, that is, reproducibility. It is also important that these readings cover a sufficient linear scan length to be representative of the sample. The stage speed, 
acquisition rate, and number of readings will determine this length. Trial and error has shown that a minimum of 12 centimeters of surface must be covered.

The second consideration is the overall system "resolution". This involves the size of the spot on the surface that is measured and the accuracy of that measurement. Together these parameters determine system resolution, that is, the ability to discriminate among species according to reflectivity.

Discrimination can be maximized within the limitations of the equipment. The factors that affect the discriminating ability of the system are discussed in the following paragraphs:

1. As the size of the spot that is measured decreases, the reading becomes more likely to describe an individual particle. However, if the size of the spot is larger than a particle, the reading will not describe an individual particle but rather a mixture of two or more particles. Therefore it is desireable to operate with as small an aperture as possible to maximize the discrimination of particles on the surface.

The intensity of light reaching the photomultiplier tube is directly dependent on the size of the spot that is measured. If the size of the spot is less than 4 square micrometers the photon flux is not sufficient to get an accurate reading (17) within the time span of a measurement. Therefore the ability to discriminate particles on the surface is limited by the apparatus to particles greater than or equal to 4 square micrometers. 
2. The accuracy of each reflectance reading is dependent on stage speed and system "noise". Together these factors effect the ability to discriminate among species by reflectivity.

Noise from fluctuations in the light source and from the associated electronic apparatus contribute only a small amount of error to any reading. The major contribution arises from the statistical nature of light. Since the stage is in continuous motion, the number of photons per sampling area entering the photomultiplier tube will decrease as the stage speed increases. That is, a counting error will be observed proportional to the scan speed. This results in a speed-accuracy tradeoff and thus limits the speed at which the sample can be scanned with a given accuracy.

3. The final reflectance reading is also dependent on the precision of the analog-to-digital converter $(A D C)$. This is one equipment parameter which can be easily modified. The present system uses an $A D C$ which resolves the photonultiplier signal to a precision of 0.0078 volts. For reflectance ranges greater than 0.0 to $4.0 \%$ extended precision may be required. However, increasing the precision of the $A O C$ cannot compensate for the inherent error in a reading due to equipment limitations. Therefore too high a precision only makes the analysis more time consuming by collecting data that yields no more information than that obtained by less precise measurement of the photomultiplier tube signal.

To summarize the limitations the above considerations impose on the experiment: 
1. The physical size of particles that can be measured is greater than or equal to 4 square micrometers.

2. The statistical nature of light does not allow discrete reflectance values to be obtained for homogeneous surfaces. Although sampling error can be decreased by signal averaging and integration, the small aperture size causes a distribution of reflectance values to be observed even for surfaces that are homogeneous.

3. Increasing scan speed decreases accuracy of a reading.

4. The linear scan length and the total population of readings determines reproducibility.

The optimum conditions chosen for this investigation are as follows: the run has a total of 102,400 readings, the stage speed is 800 micrometers/second, and the aperture size is 4 square micrometers. Therefore an acquisition rate of 400 readings/second will take about 5 minutes and cover 20 centimeters of surface. Trial of other parameters failed to significantly increase the ability to discriminate among species. Therefore these conditions represent a good combination to obtain maximum discrimination and speed of analysis for this study.

\subsection{TREATMENT OF THE DATA}

A series of reflectance readings as they would be received by the microcoliputer are shown in Figurc 2. The data stream represents a linear reflectance map of the pellet surface. The complex nature of the surface is readily apparent. Particles of different reflectance 




Figure 2. DATA STREAM OF PSOC-328 
and length, particles imbedded in each other, particles submerged in the matrix, as well as cracks in particles are all recognizable as variations in reflectivity with time (scan length).

One way to process this data stream to reduce it to a more concise and interpretable form is to construct a histogram, or reflectogram. In other words, plot frequency of occurrence of a reading versus percent reflectance. Such a plot is shown in Figure 3. Treating the data in this way will allow readings of similar reflectance, that is, similar particles, to be identified as distributions, or peaks. The prominence of a distribution should be proportional to the concentration of that species, however, the presence of particles that vary widely in size as well as reflectance, and other factors, make such a prediction inaccurate.

There are two dominant peaks that characterize a reflectogram. The first peak, in the low reflectance range, from about 0.0 to 0.2 percent reflectance, is due to the epoxy matrix. The second peak is due to the vitrinite component in the coal. This peak is prominent since vitrinite is in large concentration in most coals. The area between these two peaks is due to exinites and edge boundaries between particles. The high reflecting macerals such as fusinite and micrinite appear on the high side of the vitrinite peak. Edge readings will also populate this region.

\subsection{VERIFICATION OF TIIE DATA ACQUISITION SYSTEM}

Figure 4 shows the excellent reproducibility obtained by the previously stated standard conditions. As a further overall system check, a simultaneous acquisition using two independent computers, 


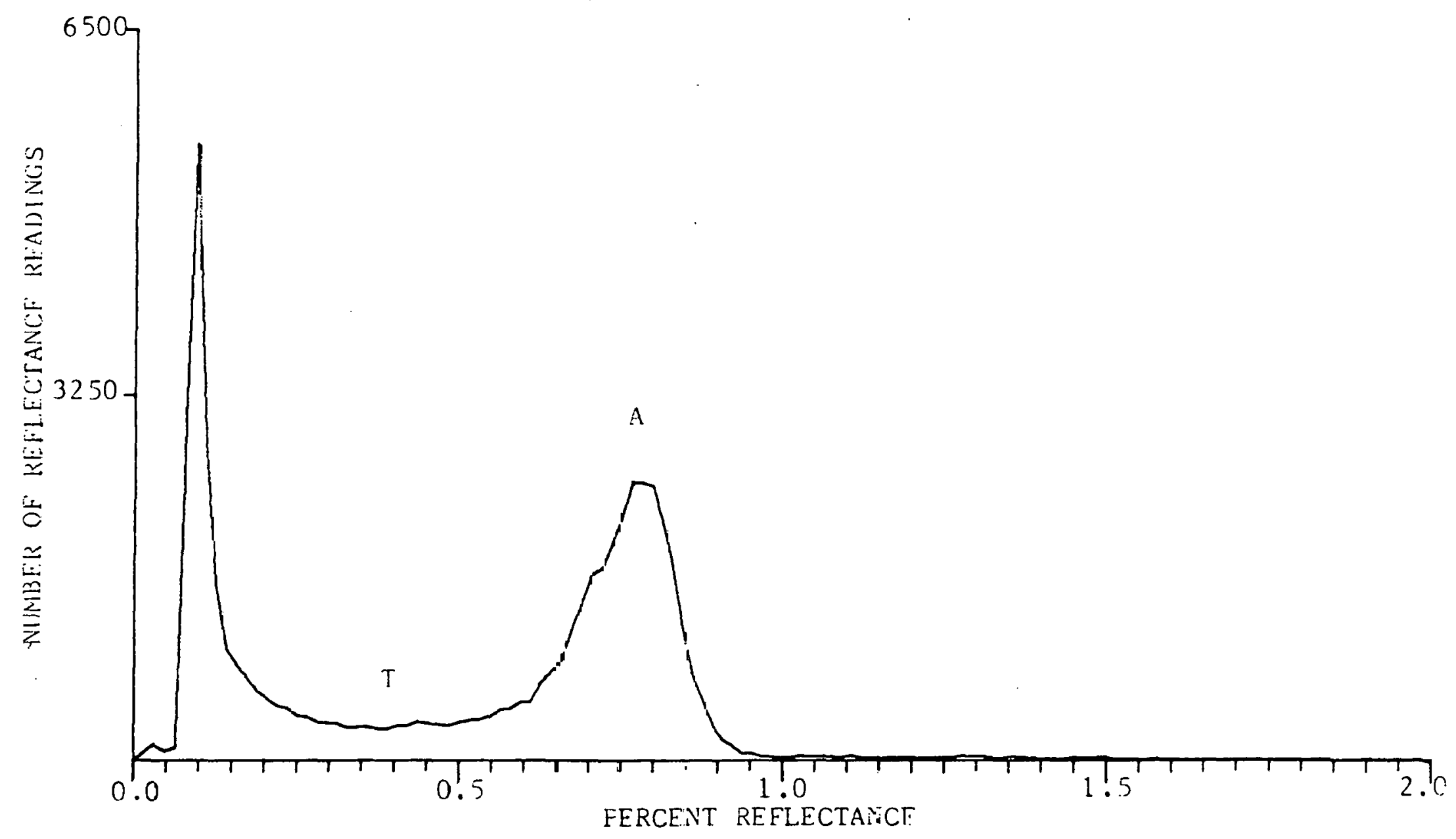

Figure 3. A REFLECTOGRAM DF PSOC-328 




Figure 4. THE REPRODUCIBILITY OF A REFLECTOGRAM (PSOC-351) 
analog-lo-digital converters, and software operating systems was made using identical run parameters. The results are shown in Figure 5. The only significant difference in the two systems was the resolution of the photomultiplier tube signal. That is, the $A D C$ in the comparison system had a precision of $0.004 \%$ reflectance and that in the system used for this study a precision of $0.016 \%$ reflectance. As can be seen, the two runs are essentially identical. 

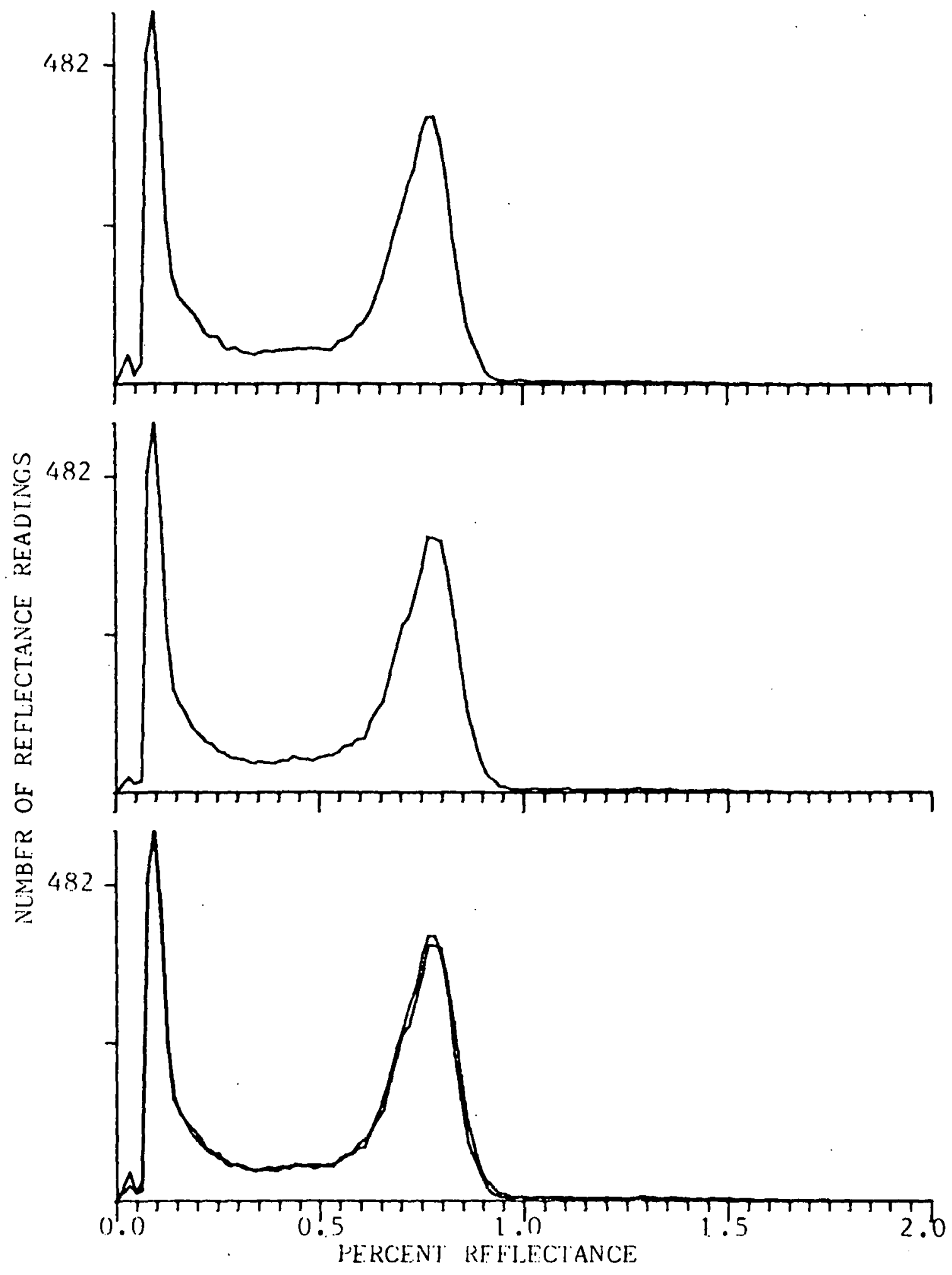

Figure 5. REFLECTOGRAMS OF PSOC-328 ACQUIRED SIMULTANEOUSLY ON A PDP-8 SYSTEM, THE KIM-1 SYSTEM, AND BOTH SUPERIMPOSED (TOP TO BOTTOM) 


\section{RESULTS AND DISCUSSION}

\subsection{INTERPRETATION OF THE REFLECTOGRAM}

To be able to discuss the reflectogram generated by the ARM system, it is necessary to understand the details of its construction. During the analysis the surface is continuously scanned. The reflectance values are recorded for consecutive spots on the surface. These are arranged and plotted as the frequency of occurrence of a reading versus percent reflectance. Therefore particles of similar reflectivity will be grouped together. However, several factors, in addition to those due to the equipment, limit the ability to discriminate among species using this method of data collection.

One limitation is that particle types are distributions. This is unavoidable for two reasons: First, coal is a birefringent material. Surfaces parallel to the bedding plane have maximum reflectance. Since a pellet is a statistical sampling of coal particles, a random orientation of particles will exist on the polished surface. As unpolarized light is used in the analysis, the recorded reflectances for any particle type will depend on its orientation. Thus a reflectance distribution will be observed for any species. Second, an amount of error will be inherent in any reading from a number of sources due to the equipment. The previous section dealt with these limitations in detail.

Since particle types are represented as distributions, if one species population is signiticantly greater than another, or if the range of two or more distributions overlap, the information may be lost in the reflectogram representation. If overlapping distributions 
were the only factor, deconvolution could be attempted by fitting functions for the various species. However, due to interfaces between particles such mathematical manipulation is at best only approximate.

The edges of particles present the major limitation in attempting to discrininate among species using reflectance readings. Readings that include a border between two or more particles fail to describe either. However, in creating a reflectogram all information is treated equally. Therefore, instead of having discrete or slightly overlapping distributions representing each species, the edge readings will skew distributions toward each other.

The population due to edges is distributed throughout the entire reflectogram, however it is most noticeable in the region between the matrix and vitrinite peaks and on the higher reflectance side of the vitrinite peak. The effect of the edge readings is a decrease in the ability to discriminate among species. The edge population tends to obscure the distributions of the major species. In other words a reflectogram contains artifacts, that is, edge readings, that do not represent any species present in the sample. These readings make both qualitative and quantitative interpretation of a reflectogram difficult.

A good index of the ability to discriminate among species using a reflectogram is the ratio of the height of the dominant, vitrinite, peak $(A)$ to the height of the transitional valley between this peak and the matrix peak ( $T$ ) (Figure 3 ). The larger this ratio the poorer the "resolution". 


\subsection{IMPROVEMENT OF THE REFLECTOGRAM}

The stream of data received for processing is subject to limitations imposed by the equipment and by the nature of the surface. There are two approaches that can improve the species discriminating ability of the ARM system. One is. to modify the hardware and the other is through software processing.

The limiting hardware factor is the aperture size. It has been noted that the smallest usable window is 4 square micrometers. However, even coal that is only -20 mesh, that is relatively large compared to 4 square micrometers, will present problems. The natural size of many macerals, such as exinites and micrinites, is less than 2 micrometers in length. A distribution representing these macerals will never be seen even though they have a unique reflectance. Fusinite is a very friable maceral that will also be found as small particles on the order of less than 15 micrometers. Therefore even large concentrations of any of these species w1ll not result in a peak in a reflectogram.

Decreasing the spot size to allow measurement of the smaller species can only be obtained by increasing the intensity of the light source. The halogen lamps used in the present equipment have a flux of 2800 lumens. High pressure mercury and xenon lamps typically have fluxes three times greater, however controlling the stability of these lamps is difficult. A more logical source would be a laser. This and other possibilities will be discussed later. Another way to improve the ability to discriminate among species is to compensate for the inaccuracy inherent in the photomultiplier 
tube signal. In other words attempt to decrease the standard deviation associated with the reflectance distributions of various particle types. Typically this is done by extending the sampling time.

One method is to use software averaging. This involves summation of a number of independent readings in rapid succession. Only the average of these readings is recorded in the reflectogram. This eliminates some of the random error in any single reading, but timing is critical when using such a technique. Since the stage is in continuous motion, the actual area sampled by the readings will be greater than the spot size. This in itself introduces error. Therefore conversion time of the $A D C$, program execution time, and stage speed must all be coordinated to make the technique effective and minimize analysis time.

A much easier and more representative technique is to use hardware averaging. By using a dual slope $A D C$, the value returned is an exact integration of the photomultiplier signal over the entire sampling period. A more accurate reading is inherent due to the continuous accumulation during the sampling time, rather than sumation of a number of independent readings. Integration time is typically shorter than that required to process a suitable number of independent readings. This technique is thus more accurate and efficient than software averaging.

Without major equipment modifications little else can be done to improve the species discriminating ability of the system from a hardware standpoint. Also even with improvement many of the problems due to the nature of the surface will still exist. 


\subsection{DATA PROCESSING TECHNIQUES}

In the previous sections, several limitations of the equipment and technique were discussed. The intent of this section is to examine software techniques that can be executed in real time to process the incoming data. Hopefully the utilization of data processing techniques will increase the amount of information obtained from a run and thus enable more precise characterization of the sample. At the very least, insight into how various parameters effect the generation of a refiectogram will be obtained.

The concept of changing the data stream to a reflectogram was discussed in section 3.5. A reflectogram is only used to present the overall statistics in an easily recognizable form. The additional information that was available as time-history will disappear in such a presentation, therefore any processing will have to be performed on the data stream before the reflectogram is constructed.

The information that is in the data stream can be used to determine which readings are coal, matrix, edges, what is a particle's length, and of course the percent reflectance. The problem is to devise an algorithm to extract as much of this information as possibie. When using an algorithm to modify data, it is possible to distort information due to the algorithm itself. For software processing to be useful it must utilizc a criteria that in some way enhances the original data without distorting the final results. One approach is to attempt to use criteria to eliminate what are thought to be artifacts in the original data. Such a technique only rejects (eliminates) certain data from the original set, therefore by comparing the original 
data and the processed (transformed) data, judgement as to the validity of the transform can be made.

It is essential to be able to describe what a particular transform does and to evaluate its success or degree of discrimination. To do this a presentation scheme has been adopted that consists of the following four plots: (I) the original reflectogram (standard conditions), (II) the transformed reflectogram, (III) the original minus the transform, (IV) the ratio of the original to the transform. Figure 6 shows such a series of plots. The origin of plot types I and II are straightforward. They represent standard conditions and the applied transform, or in other words, before and after. Plot type III will show how the two reflectograms differ, that is what has been eliminated due to the transform. Plot type IV will show the percentage change generated by the transform. Thus in viewing a set of these plots a good idea of the selection criteria of a particular transform will be obtained.

\subsection{DATA ANALYSIS USING A CONNECTIVITY ALGORITHM}

The data stream of a coal pellet is ideally suited to a simple processing technique that uses connectivity as the distinguishing criteria. It has been previously discussed why edge readings are a major limitation in allowing a reflectogram presentation to discriminate among species. By using the time-history information available in the data strean an edge reading can be letected and eliminated by measuring connectivity. Therefore it is expected that a transform based on connectivity will increase the ability to discriminate among 


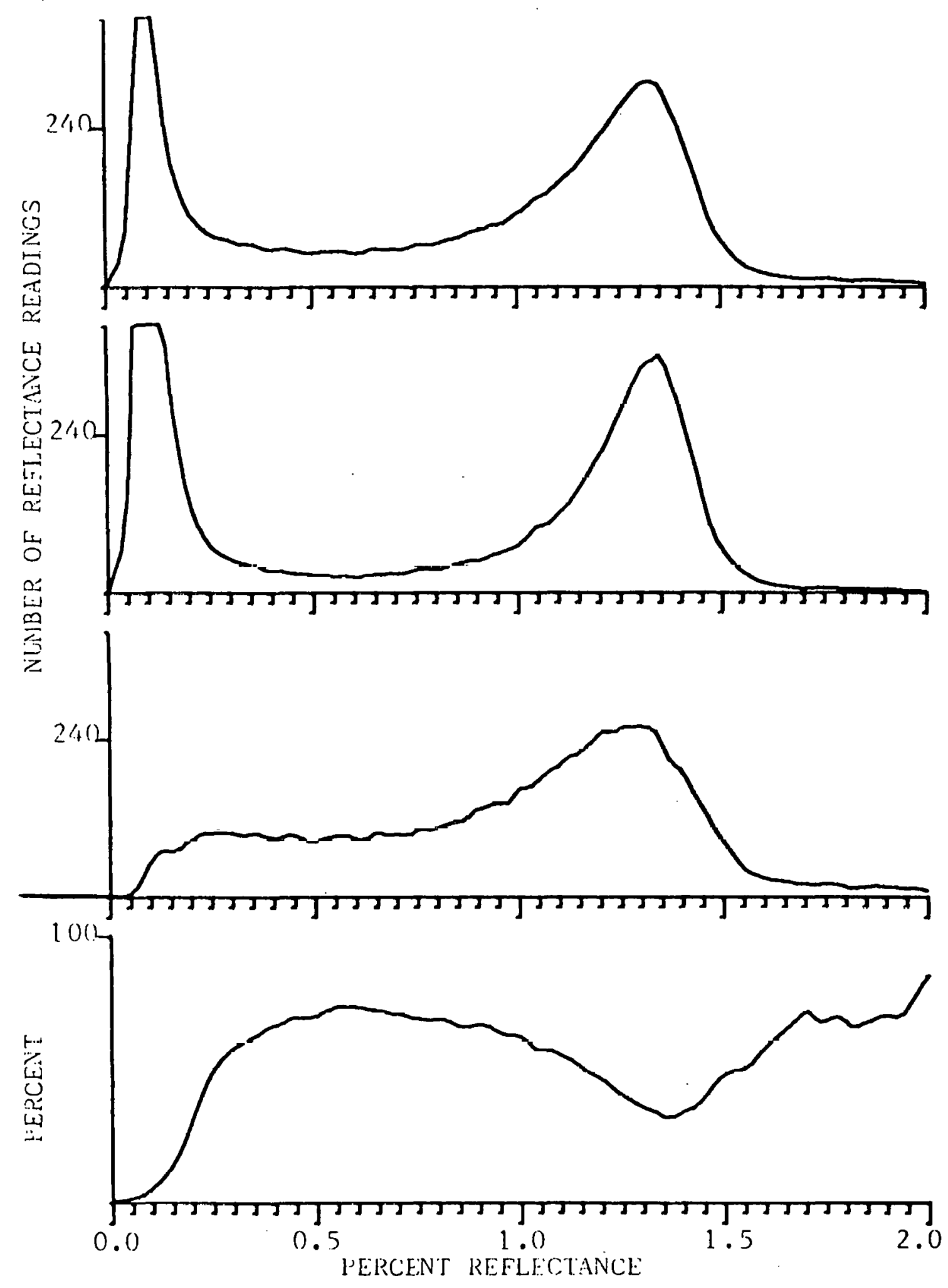

Figure 6. A SERIES OF REFLECTOGRAMS OF PLOT TYPE I, II, III AND IV (TOP TO BOTTOM) 
species using a reflectogram as well as yield information on the particle size distribution of the sample.

A pellet consists of a number of different-sized particles, or. discrete homogeneous species. To attempt to measure connectivity, a nearest neighbor, or threshold technique, can be used. The flow chart of the algorithm is given in Appendix C. Each successive data point, representing consecutive 4 square micrometer spots on the pellet surface, is compared to the preceeding point. Those values within a fixed reflectivity range are considered to be on the same particle, that is, connected. Using a reflectance range of $0.09 \%$ allows discontinuities to be distinguished without rejecting readings from a homogeneous surface. By counting the number of consecutive hits within the reflectance range, each representing a length of 2 micrometers, a connectivity length is determined. This technique can be enhanced by simultaneously constructing many reflectograms while processing the data, each representing a different connectivity length. Therefore, from a series of reflectograms acquired during a single run the effect of connectivity on a reflectogram can be observed.

Figure 7 presents a series of reflectograms each representing a different connectivity length. The largest population is for lengths of greater than or equal to 2 micrometers, that is, the aperture size. This distribution contains all the data that was received. The next largest population is for particle sizes greater than or equal to 4 micrometers, that is, two consecutive aperture sizes have readings within the specified reflectance range. This plot represents data that has been filtered according to the connectivity criteria. The 



Figure 7. A SERIES OF REFLECTOGRAMS REPRESENTING PARTICLE SIZES OF GREATER THAN OR EQUAL TO 2, 4, 8 AND 16 (TOP TO BOTTOM) 
effect is seen as an increase in the ability to distinguish between species. Some of the data was eliminated, and through this process information can be inferred about the surface. The other two plots are for particle sizes of greater than or equal to 8 and 16 micrometers. They show an even greater increase in distinguishing ability. The population of a particular connectivity length can be obtained by taking the difference between any two distributions. For example:

population $\geq 2 u m-$ population $\geq 4 u m=$ population $<4 u m$ This is plot type III as described in section 4.3. A series of plots of this type are shown in Figure 8. These plots are ail of equal population and represent particle sizes of $<4,{ }^{\circ}<8,<16$, and $<32$ nicrometers.

The selectivity with respect to length is more readily apparent in this series of plots. Notice that in the plot of lengths less than 4 micrometers a significant population occurs in the region between the peaks of the two prominent distributions. This region, between the low reflecting matrix material and the higher reflecting vitrinite in the coal, can be referred to as the transition region. The only macerals with reflectance readings in this range are exinites, which are typically a small percentage of the coal compared to the population of this region indicated by the reflectogram. The bulk of the observed population can be attributed to matrix/coal edge effects. Apparently then, Figure 8 shows that edges are being eliminated by utilizing a length criteria. Proceeding to the plots of longer length it is seen that more and more of the vitrinite and 

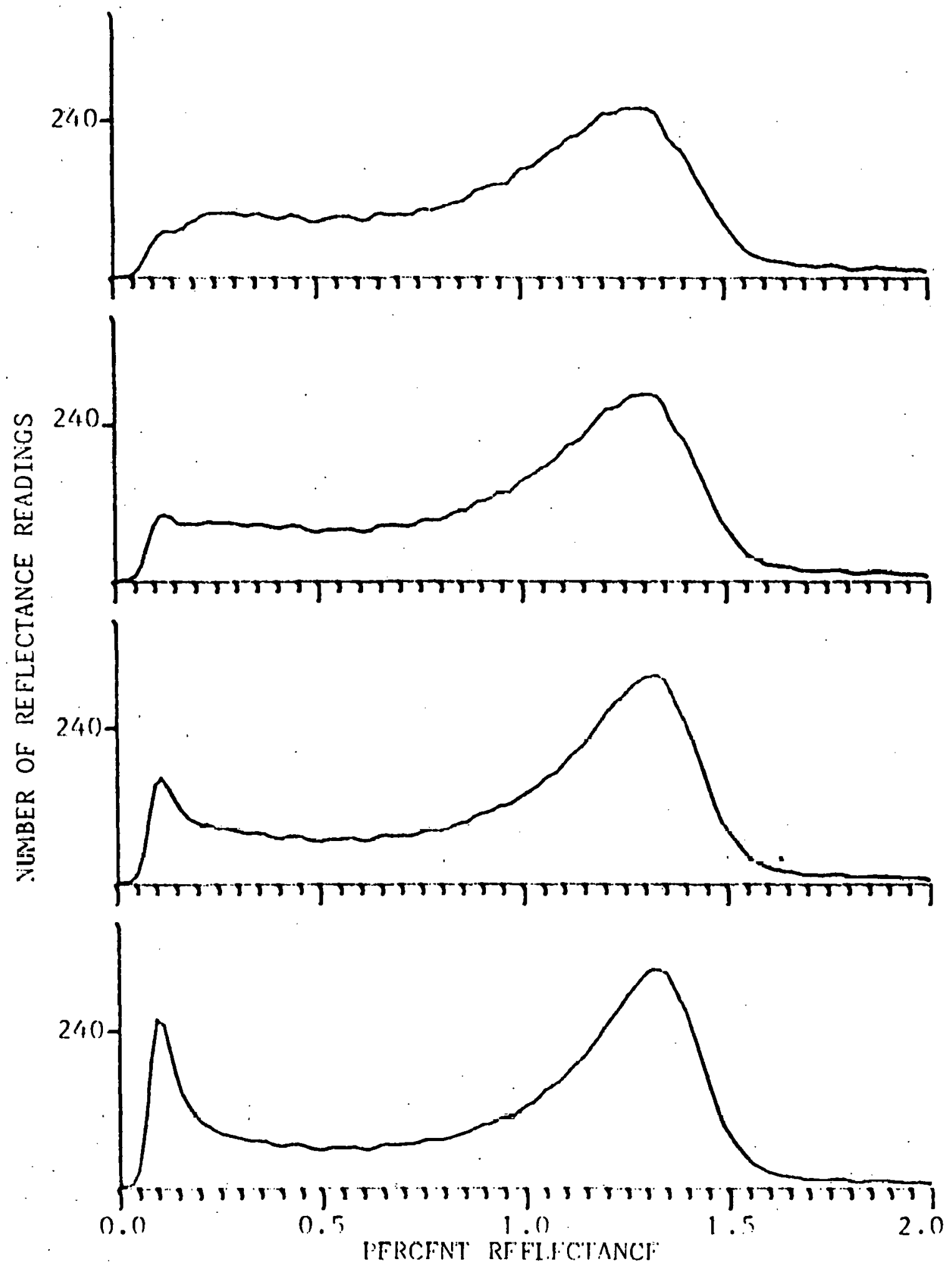

Figure 8. A SERIES OF REFLECTOGRAMS REPRESENTING PARTICLE SIZES LESS THAN 4, 8, 16 AND 32 MICROMETERS (TOP TO BOTTOM) 
and matrix distributions, that is, larger particles, begin to be el ininated, as expected.

A series of type IV plots are shown in Figure 9. These show the percent change generated by the transform. Again regions known to arise from matrix/coal edges are eliminated to a greater extent than those from the primary distributions. This series also shows that the criteria are no longer selective at longer lengths.

What conclusions can be drawn from the described connectivity discrimination transform? The technique increases the ability of a reflectogram to discriminate among species by the elimination. of readings in regions known to arise from edges and small particles. In other words, interfaces between particles, known to be artifacts and not indicative of surface composition, as well as small particles whose reflectance is incorrectly measured, are eliminated by processing.

This treatment allows several observations. First, the largest particles in a pellet are matrix, since the area of this distribution decreases more slowly with increasing connectivity than the vitrinite distribution (Figure 7). Second, there are very few large homogeneous coal particles. Typically 50\% of the total population gathered with no discrimination ( $\geq 2 \mathrm{um}$ ) is eliminated by the greater than or equal to 4 micrometer length criteria, meaning that the coal particles are snial1. A plot of the actual data stream as shown in Figure 2 substantiates that the observed particle sizes are generally small given the particle size (850 micrometer maximum) of the coal used to form the pellets. In other words a greater population of larger coal particles would be expected. 


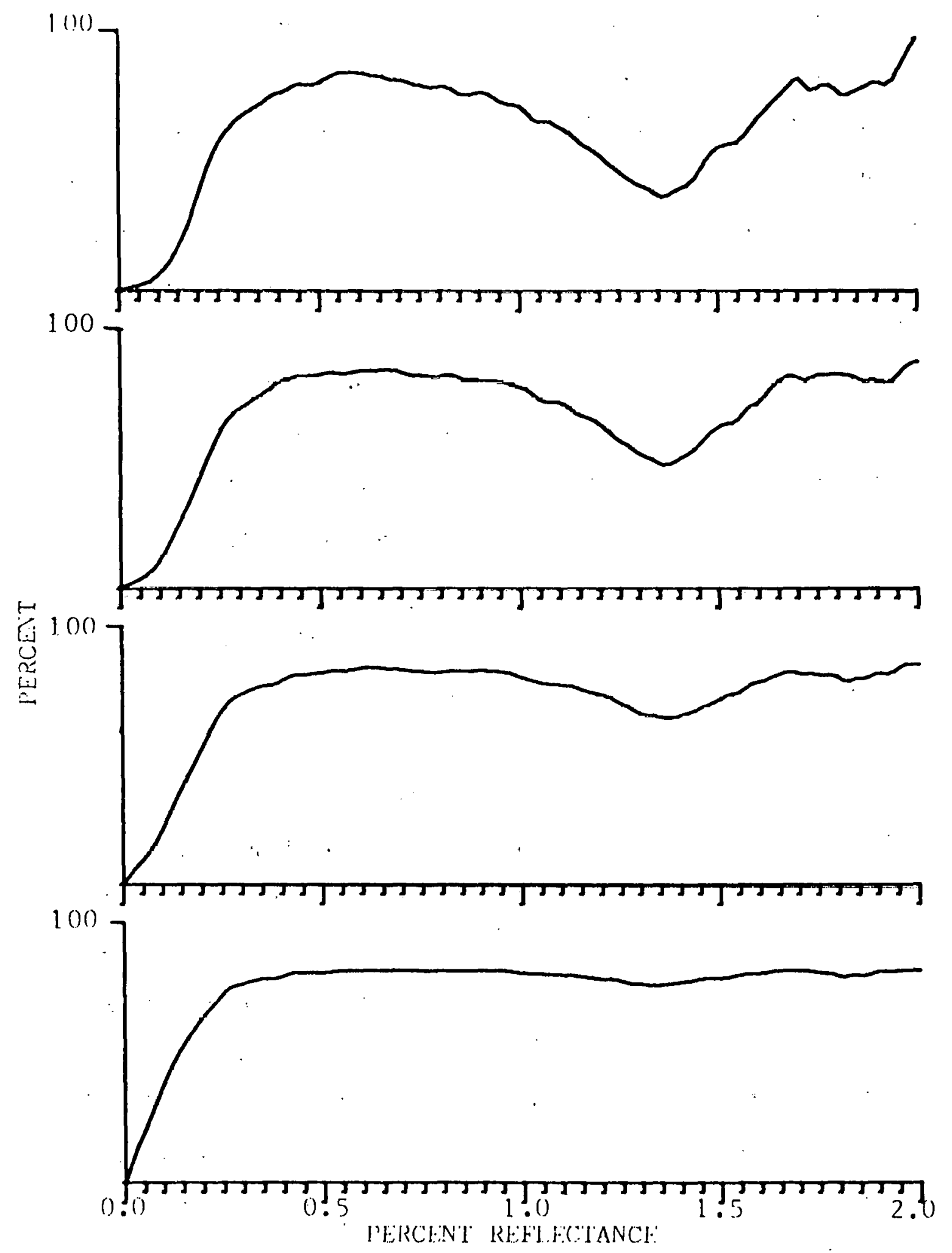

Figure 9. A SERIES OF REFLECTOGRAMS SHOWING THE PERCENTAGE CHANGE BETWEEN PARTICLE SIZES OF $\geq 2$ MICROMETERS AND PARTICLE SIZES OF $\geq 4, \geq 8, \geq 16$ AND $\geq 32$ MICROMETERS 
A close look at individual particles reveals why so many interfaces are observed. Figure 10 shows the data stream of a pellet with a $5 \%$ concentration of coal. The individual coal particles can now be easily distinguished. The microstructure within a particle is graphically shown. Where as from Figure 2 it appears there are many "cracks" within particles, it would now seem that these are not so much fractures filled in by epoxy, but small particles closely packed. The inhomogeneity of a single particle is apparent in Figure 10, but what is very significant is that true particle edges, interfaces between matrix and coal, are sharply defined. Very few such interfaces are found in the data stream representing a pellet that is $80 \%$ coal (Figure 2), suggesting that close packing is creating a distribution of matrix/coal interfaces.

In pellets which are $80 \%$ coal the coal particles are closely packed. This close packing means that the boundaries between particles will be filled in by only shallow layers of epoxy. Figures 2 and 10 show that the degree of packing, that is, the concentration of coal in the pellet, strongly effects the number of readings that are due to measurement.s on surfaces submerged in the matrix.

Epoxy is much less absorbing than coal, thus measurements can be made on coal surfaces which are slightly submerged in epoxy. The reflectivity of these surfaces will depend on the depth of the particle beneath the pellet surface. Measurement of these surfaces are not descriptive of any species, coal or matrix. They are essentially a "type of edge" in that they only skew the reflectance distribution of other species. Clearly the number of these types of edges increase with the concentration of coal in a pellet. 




Figure 10. DATA STREAM OF A PELLET WITH A LOW CONCENTRATION OF COAL OF COAL 
To attempt to further substantiate the change in the data stream caused by changing the concentration of coal in the pellet, a simple geometric model can be used. The details of this argument will be elaborated later, but essentially the argument can be used to show that the expected number of volumetric edge readings (matrix/coal interfaces) is a relatively small percentage of the total population of readings for most particle sizes (particularly -20 mesh).

The high number of total edge readings observed (as indicated by the area of the transition region) must therefore arise from the microstructure within particles and the "edges" due to packing. Figure 10 would suggest that pellets with a smaller concentration of coal would benefit the analysis by allowing matrix/coal interfaces to be eliminated by simple software processing. The ability to discriminate between species using a reflectogram would be enhanced significantly with the elimination of volumetric (matrix/coal) edges and the decrease in the standard deviation of the matrix distribution.

\subsection{BIMODAL SAMPLES}

To further evaluate the effect of particle size on a reflectogram and examine the possiblity of determining the microparticle size distribution of a pellet, a series of "synthetic" coal pellets were made. Two coals of equal rank and approximately equal petrographic composition but different mean/maximum vitrinite reflectance were chosen from the Penn State/DOE Coal Data Base and combined to generate a bimodal distribution. The pellets are composed of $40 \%$ by volume of each coal and $20 \%$ epoxy, however the particle size of one coal was varied. Data concerning the series is outlined in Table 1. 
Table 1. Composition of Synthetic Pellets Used to Examine the Connectivity Algorithm

Data from the Penn State/DOE Coal Data Base:

COAL A

PSOC Number

Mean/Maximum

Vitrinite

Reflectance

\% Exinite (by vol., DMMF)

$\%$ Vitrinite (by vol., DMMF)

\% Fusinite (by vol., DMMF)

\% Mirrinite (hy vnl., nMMF)

102

0.84

3.9

82.5

8.4

5.2
Composition of Pellets:

Pellet Number

1

2

3

4

5

$$
\begin{gathered}
\text { Particle size } \\
\text { (IIS Mesh) } \\
\text { COAL A } \\
\hline
\end{gathered}
$$

$50 \times 60$

$20 \times 35$

$60 \times 80$

$80 \times 100$

$20 \times 35$
COAL B

409

1.65

90.0

8.5

1.5
Particle Size (IIS Mash) COAL B

$50 \times 60$

- $50 \times 60$

$50 \times 60$

$50 \times 60$

$35 \times 50$ 
Figure 11 is a plot of a run (standard conditions) on pellet number 1 , that is equal particle size for each coal. As expected three prominent distributions are observed. One for the matrix and one for the vitrinite in each coal. At first glance it would appear that the coals are not in equal percentage by volume, as the area of coal $A$ is about twice that of coal B. This illustrates quite nicely how distributions interact with each other.

The greater the distance between the mean reflectivity of the distributions, the less they distort one another. The number of edges contributing to the transition region population is the same regardless of the distance between the distributions, but moving the distributions apart increases the reflectance range of the transitional readings. Thus as the number of reflectance catagories between the coal and matrix increase, edge readings become less apt to fall close to either major distribution.

Figure 12 illustrates this effect by showing a series of plots for increasing rank of coals. Notice as rank increases, the area of the matrix decreases, due to transitional readings spreading away from the true distribution. The apparent standard deviation of the matrix distribution thus decreases. The same is true for coal, although less obvious since the degree of bireflectance increases with rank.

To show unambiguously that pellet number 1 (and the others in the series) is an equal volume mix of each coal, a run on a pellet of PSOC-102 and PSOC-409 was made. These independent runs were then surmed to produce a bimodal distribution. Figure 13 proves that indeed pellet number 1 is an equal volume mixture of coal $A$ and $B$. 


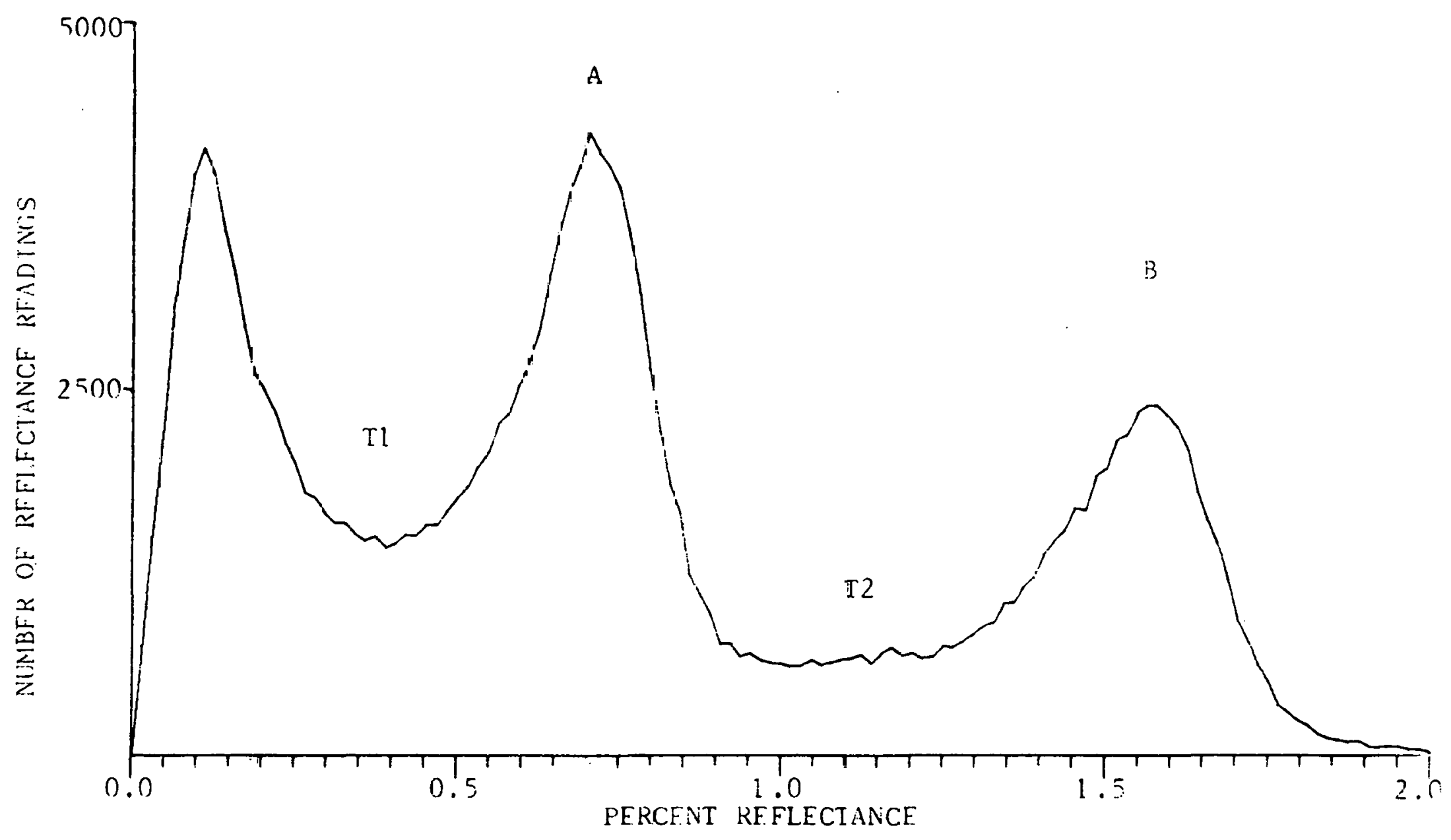

Figure 11. REFLECTOGRAM OF PELLET NUMBER 1 


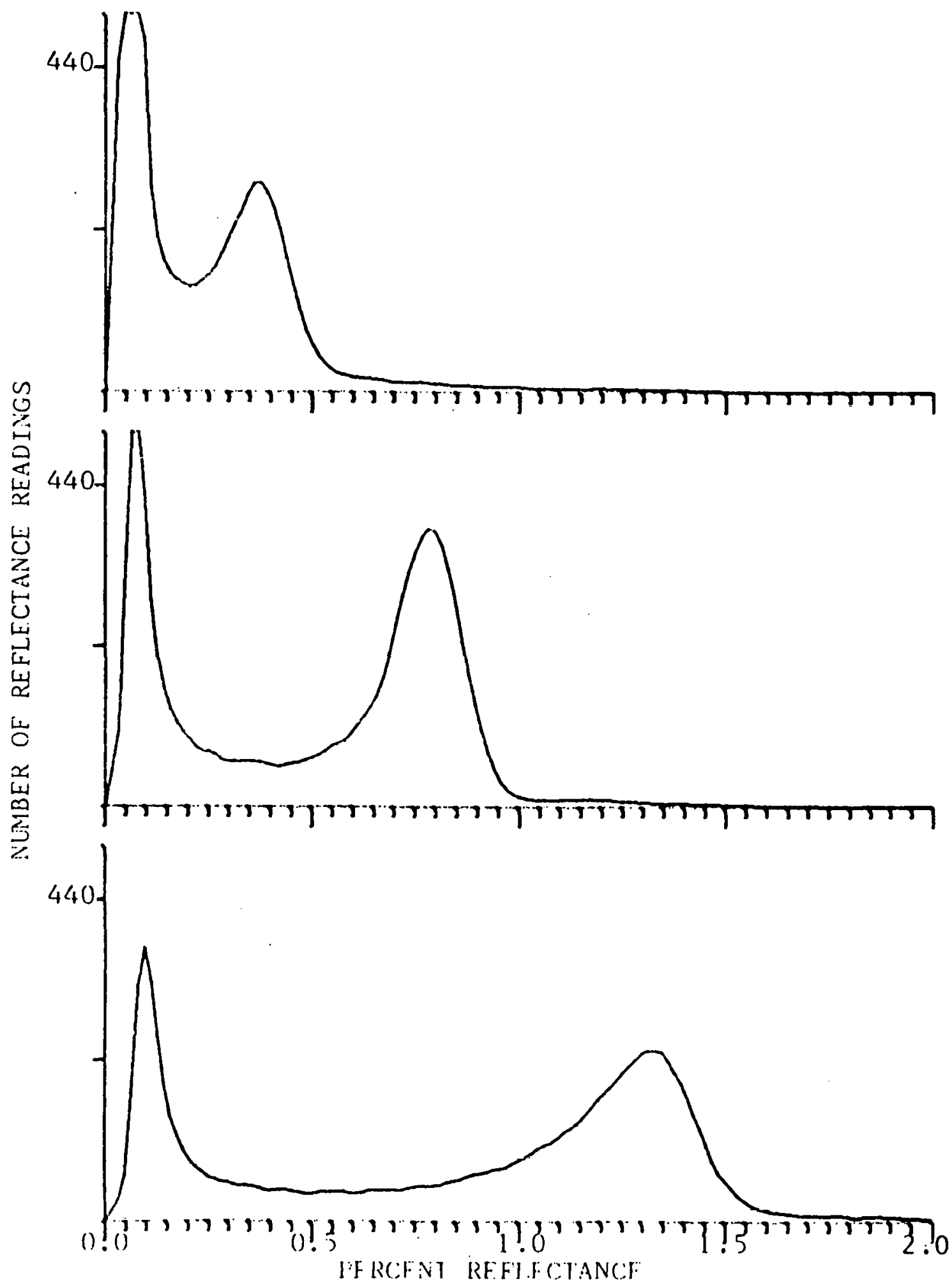

Figure 12. A SERIES OF REFLECTOGRAMS FOR COALS OF INCREASING RANK (TOP TO BOTTOM) 




Figure 13. TOP TO BOTTOM: PSOC-102, PSOC-409, PSOC-102 + PSOC-409 AND PELLET NUMBER 1 
The bimodal experiment was designed so that coal $B$ would be an internal standard, that is remain constant as the particle size of coal A was varied. In this way the effect of particle size could be readily examined. Figure 14 is a series of three plots all of equal population representing pellets number 2,1 , and 4 . In other words from top to bottom, coal A changes in particle size from larger than $B$, to equal to $B$, to smaller than $B$, while $B$ remains constant.

This series illustrates several points. First, from the increase in the ratio of the peak of $A$ to the transition valley (Tl) it is apparent that the ability to discriminate among species is decreasing with decreasing particle size. This is to be expected from the previous discussion. If the curves were normalized to the height of $B$, it would be apparent that the height of $A$ is decreasing with particle size, also indicating decreasing ability to discriminate among species.

Second, something can be said about the types of edges that cause the observed decrease in the ability to discriminate among species. If epoxy tends to surround coal particles, then as particle size decreases the number of observed volumetric edges due to this interface should increase. The assumption based on Figure 14 is for this type of behavior, that is, that only the number of matrix/coal interfaces should increase by physically changing particle size. The microstructure of the coal, due to inhomogeneity from maceral and mineral matter inclusions, and pitting of the surface will be essentially unaffected by particle size. 


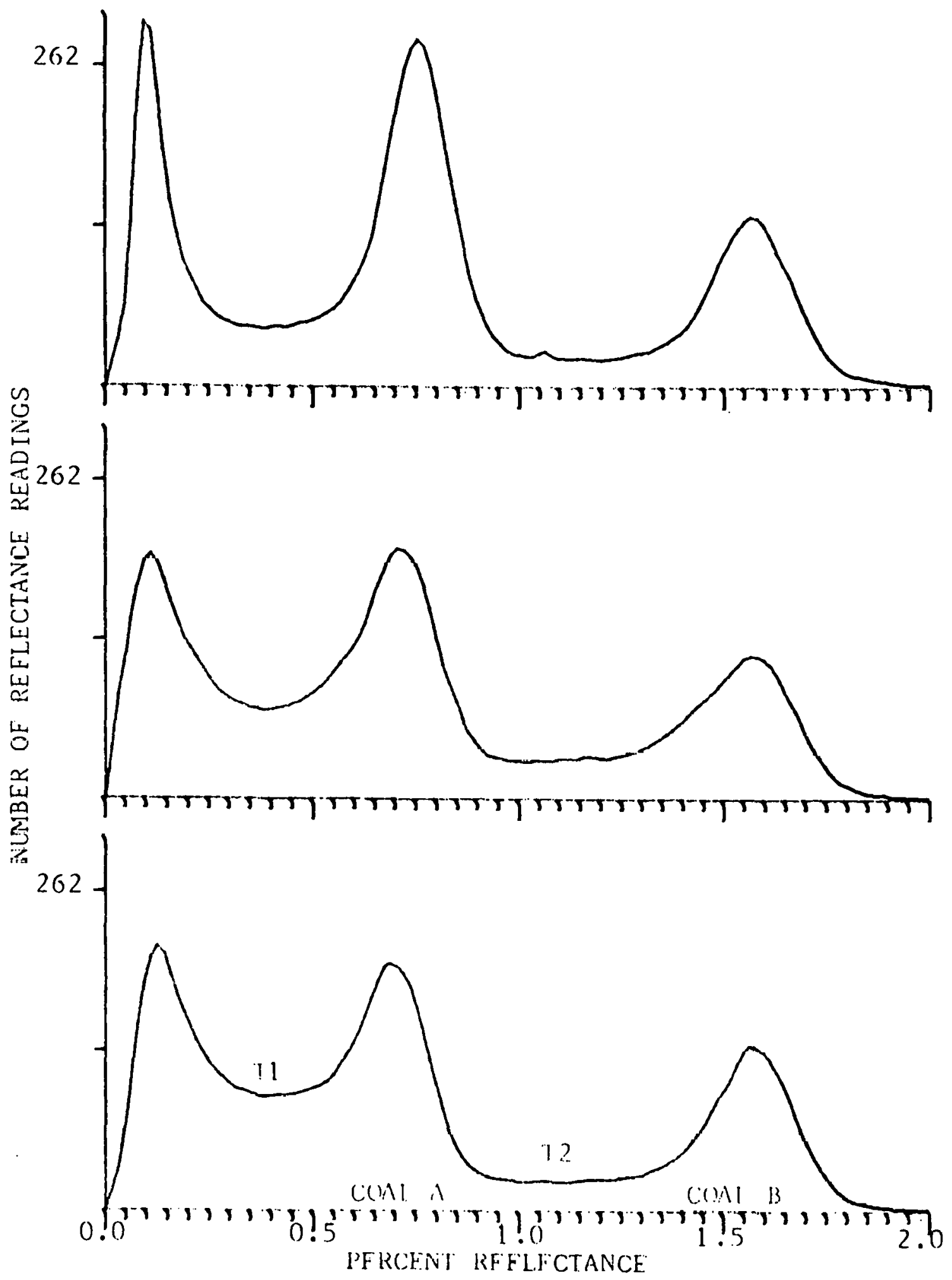

Figure 14. REFLECTOGRAMS OF PELLETS NUMBER 2, 1 AND 4 (TOP TO BOTTOM) 
Using the data from the bimodal pellets a calculation can be made to show that this assumption is sound. Assuming spheres for particle shape, the probability of a reading representing an edge transition for a given particle radius, $R_{0}$, for a given total volume of sample can be calculated as follows:

If $\Delta$ equals the aperture size, then any reading taken within $R_{0}+\Delta$ to $R_{0}-\Delta$ represents an edge. The probability of getting a reading in this region is the ratio of the volume of a shell from $R_{0}+\Delta$ to $R_{0}-\Delta$, to the volume of the sphere itself.



$$
\begin{aligned}
& P_{\text {edge }}=\frac{\text { volume of shell }}{\text { volume of sphere }}=\frac{\frac{4}{3} \pi\left(R_{0}+\Delta\right)^{3}-\frac{4}{3} \pi\left(R_{0}-\Delta\right)^{3}}{\frac{4}{3} \pi R_{0}^{3}} \\
& P_{\text {edge }}=\frac{R_{1}^{3}=R_{2}^{3}}{R_{0}^{3}}
\end{aligned}
$$

The calculated percentage of volumetric edges versus particle size arc shown in Table 2. To obtain this percentage an aperture size of 1 microneter $(\Delta)$ was used in equation I instead of the 2 micrometer aperture size actually used in the experiment. A choice of 1 micrometer is necessary because of the threshold technique used to detect edges. A transition edge was defined in 
Table 2. Calculation of the Expected Amount of Microstructure Using a Simple Spherical Model.

\begin{tabular}{lcccc}
\hline Pellet & $\begin{array}{c}\text { Mean Volume } \\
\text { Diameter } \\
\text { Number }\end{array}$ & $\begin{array}{c}\text { Calculated } \\
\mathrm{E}_{2}\end{array}$ & $\begin{array}{c}\text { Measured } \\
\mathrm{E}_{1}\end{array}$ & $\begin{array}{c}\text { Microstructure } \\
\mathrm{E}_{2}-\mathrm{E}_{1}\end{array}$ \\
\hline 2 & 718 & 0.84 & 3.40 & 2.56 \\
1 & 277 & 2.17 & 5.22 & 3.05 \\
4 & 166 & 3.61 & 6.53 & 2.92 \\
\hline
\end{tabular}


the connectivity algorithm to be a change in reflectance of greater than $0.09 \%$, but typical matrix/coal interfaces only change from 0.1 to $0.8 \%$ reflectance in these pellets. Therefore it would be necessary for the aperture to fall almost exactly in the middle of every transition region in order to detect every reading that represents an edge. Decreasing the aperture size to 1 micrometer in equation I accounts for the fact that not all readings representing transition edges are detected by this technique.

Table 2 also shows the percentage of edge transitions measured during a run. These values were obtained by measuring the mid-range population of $\mathrm{Tl}$ in the appropriate reflectogram. The difference between the calculated and measured values is attributed to microstructure.

The percentage of readings due to the microstructure is relatively constant, even with the assumption of spheres for particle shape and the crude approximation of the number of edges. Physically increasing the particle size will decrease the number of volumetric edges. Conversely, edges will increase rapidly with decreasing particle size as is seen, and in fact almost entirely account for the loss of resolution observed.

Briefly then, it has been shown that matrix/coal interfaces are a major cause of loss of the ability to discriminate among species using a reflectogram. These interfaces are strongly size denendent.. Two ways to minimize this loss are to lower the concentration of coal in a pellet and to increase the particle size of the coal. In either case surface heterogeneity will remain relatively constant. 
The connectivity algorithm can also be applied to these pellets as shown in Figure 15. Essentially the algorithm rejects particles less than a given length. This is similar to physically changing the particle size except that inhomogeneity within particles will be rejected in addition to volumetric edges. The same edge probability calculation as in Table 2 shows that removal of only volumetric edges cannot account for all of the points that are eliminated as the connection length criteria increases. This is only logical as much of the surface microstructure will look like small particles and be eliminated. The ability to discriminate among species will increase with the elimination of particle inhomogeneity in addition to volumetric edges.

With the increased resolution the algorithm generates, detection of other inacerals would be possible is they were of sufficient size. The problem is that the most probable chord lengths for a given diameter sphere are much less than its diameter. For any given sphere, the probability of measuring a chord length equal to the diameter of the sphere is quite small. But the probability of measuring a chord length less than the diameter of the sphere is large. In fact the probability of measuring any given chord length increases as the length of that chord decreases, that is, the probability of measuring a chord of length equal to $\Delta R \propto(R-\Delta R) / R$, where $R$ equals the radius of the sphere. Therefore it is much more likely that a chord length will be measured for a given particle size that is much less than the particle's mean diameter. This explains why the observed chord lengths in Figures 2 and 10 are small for a coal that is -20 mesh. 




Figure 15. REFLECTOGRAMS REPRESENTING PELLET NUMBER 1 AT PARTICLE SIZES OF GREATER THAN OR EQUAL TO 2, 4, 8, AND 16 MICROMETERS (TOP TO BOTTOM) 
It has been already stated that macerals other than vitrinite are typically in concentrations of less than $30 \%$ by volume and relatively small in size. Thus these two natural factors, when combined with the minimum size that can be measured by the equipment and the high probability of measuring a chord length much less than the particle diameter, make semi-quantitative detection difficult.

Figure 16 shows an attempt at semi-quantitative detection using a sample that has been subjected to fractionation and has a fusinite concentration of $54 \%$ by volume, vitrinite $41 \%$. The high concentration of fusinite is evidenced by the substantial area of high reflectivity on the right side of the vitrinite peak. The ability to discriminate among species is drastically increased by the transform, but the readings attributed to fusinite are immediately eliminated due to the small size of the fusinite particles. Therefore although a high concentration of fusinite is present and algorithmic enhancement is significant, the aperture size limitation is difficult to overcome.

Besides increasing the ability to discriminate among species, the connectivity algorithm generates data that can be used to obtain a particle size distribution. How can a particle size distribution be obtained from connectivity data? Although each particle itself will have a chord length distribution, a simple spherical model can be used to calculate the number of readings at a given chord length to be obtained for spheres of any given diameter. For spheres of equal dianeter (monosized) this is a linear relationship. In a collection of differently sized spheres the population of a particular chord length will contain contributions from all spheres 




Figure 16. REFLECTOGRAMS OF PSOC-013, AOe-1 AT PARTICLE SIZES OF GREATER THAN OR EQUAL TO 2 AND 4 MICROMETERS AND LESS THAN 4 MICROMETERS (TOP TO BOTTOM) 
with diamelers greater than or equal to that chord length. Therefore the curve for greater than one sized sphere will not be linear. However, this curve can be deconvoluted by a simple algorithm to obtain the individual particle sizes that are contributing to it. Appendix B presents a simple example. Further discussion of stereologic transforms and deconvolution methods can be found in Dinger (19).

The example in Appendix $B$ can be generalized to any number of differently sized spheres mixed in any proportions. Deconvolution to obtain the particle size distribution is done by successive regression of straight lines on the curve. The slope of the line will deternine the diameter of the contributing sphere. The area under the line when normalized will determine the percentage of the total population contributed by that diameter, and thus the particle size distribution.

To make this technique work for coal pellets a calibration curve is needed so that the sphere assumption can be eliminated. To account for particle shape factors, curves for various mesh sizes could be used. The actual calculation would be similar, except that the deconvolution would not use lines, but rather the calibration curves made for known particle sizes. Data obtained by the connectivity algorithm could then be used to calculate a particle size distribution. 


\section{CONCLUSION}

\subsection{SUMMARY}

The potential of an Automated Reflectance Microscope (ARM) system to determine the petrographic composition of a coal has been examined. The analysis involves the automatic scanning of a polished coal surface with a reflectance microscope. The reflectivity of consecutive 4-square-micrometer spots on the surface is measured by a photomultiplier tube and recorded by a microcomputer.

The readings are assembled into a histogram of reflectance values versus their frequency of occurrence, called a reflectogram. The profile of the reflectogram is a function of the composition of the sample, that is, reflectance distributions corresponding to each different species in the sample contribute to the reflectogram.

Qualitative and quantitative interpretation of a reflectogram is fifficult because of limitations which arise from the equipment itself and from the method that is used to generate the reflectogram. The major equipment limitation is the size of the spot that can be accurately measured by the photomultiplier tube. The intensity of the light source and the sensitivity of the tube do not allow a spot having area less than a 4 square micrometers to be measured. Therefore, since some macerals naturally occur less than, or on the order of, this size, representative measurements for these species cannot: be obtained.

A reflectogram is constructed from the reflectivities of all the measurements made during a run. Since the surface is composed of many species, it is possible to take readings on spots that 
represent combinations of two or more species. These edge readings will be included in the reflectogram along with measurements on only single species. Edge readings arise from the volumetric boundaries of particles, as well as from surface microstructure, that is, cracks in particles, pitting from polishing, and inclusion of particles within each other. Edge readings are artifacts since they do not accurately describe any species in the sample. The effect of these readings on a reflectogram is to distort the reflectance distributions of the species that are measured. Therefore the ability to discriminate among the species present in the sample is decreased by measurements on edges.

This study was aimed at making the interpretation of a reflectogram more straightforward, that is, increasing the ability to discriminate among species. Although hardware improvements to decrease the spot size and the error associated with each reading would allow some increase in the ability to discriminate among species, the heterogeneous nature of the surface still limits the qualitative and quantitative information that can be derived from a reflectuyrall. Therefore a real-time data processing algorithm was implemented during data acquisition to study the effects that processing can have on a reflectogram.

Data processing to enhance the information that can be derived from an experiment depends on a selection criteria that el ininates a particular feature from the data set. In the case of a coal pellet, connertivity ran he used as a distinguishing feature. By measuring connectivity it was found that edge readings could be indirectly eliminated. This greatly improved the ability to discriminate among 
species. Further investigation of this algorithm led to the conclusion that physical particle size has a major effect on a reflectogram. This effect is as strong as that obtained by changing the petrographic composition and thus quite inportant. It was also found that besides physical particle size, the concentration of coal in a pellet has an effect on the ability to discriminate among species.

A bonus of using processing to enhance the data was the ability to simultaneously gather information on particle size distributions. It was found that not only physical particle size distributions, but also maceral size distributions should be obtainable by the proper calibration curves. Simple geometric models were used to explain how the particle size distribution calculations could be obtained from lineal data.

Based on the results presented the potential of an ARM system is improved by algorithm enhancement. The processing of the data allows some of the inherent 1 imitations in the experiment to be reduced. The possibility of increasing the ability to discriminate among species and of simultaneously obtaining many types of analyses in real-time make the ARM system a viable characterization tool.

\subsection{SUGGESTIONS FOR FUTURE RESEARCH}

Photon flux to the photomultiplier tube is a principal factor

limiting the ability to discriminate among species. To decrease the spot size a higher intensity source is needed. The obvious one is a laser. A spot size of 1 square micrometer would not be unrealistic with such a system. The possibility of replacing the expensive reflectance microscope would also exist. Video disk (20) 
reading systems are now commercially available at a very nominal cost. These systems come complete with a laser source and the necessary optics and detection devices. Such systems may possibly be applied at a cost that is 40 times less than the cost of a reflectance microscope.

This study found that the concentration of coal in a pellet can effect the interpretation of the results. Thus it would be interesting to examine this effect more closely by making a series of pellets at various concentrations of coal.

The connectivity algorithm that has been used in this study is not optimum. Improvements can be made to make it dynamic so that the increase in bireflectance with rank can be accounted for. There is much work that can be done in the entire area of algorithmic enhancement, particularly with the power of dedicated microprocessor control. The results of this study should encourage further development in the area of data processing.

The possibility of obtaining particle size distributions was discussed. Besides increasing the ability of a reflectograni to discriminate among species, the connectivity algorithm can be used to calculate both a physical particle size distribution, and a microparticle, or maceral, size distribution. Unfortunately the aperture size limitation does not permit the calculation of a maceral size distribution and the high concentration of coal in a pellet does not allow determination of a physical size distribution. However, the potential of such a powerful and yet simple processing technique should be further demonstrated. Calibration curves must be made from closely sieved samples. The proper algorithms must be written to 
deconvolute the data. The determination of particle size distributions from lineal data can be evaluated only after this work is completed. 


\section{REFERENCES}

1. McCartney, J.T., and Teichnuller, M., Fuel, 51, pp. 64-68, (1972).

2. Schapiro, N., and Gray, R.J., J. Inst. Fuel, 37, pp. 234-242 (1964).

3. Davis, A., Analytical Methods for Coal and Coal Products, (C. Karr, ed.), Academic Press, Inc., 1978, pp. 27-81.

4. Dormans, H.N.H., Huntjens, F.J., and Krevelen, D.W. van, Fuel, 36, pp. 321-339, (1957).

5. Hoffman, E., and Jenkner, A., Glueckauf, 68, pp. 81-88, (1932).

6. Huntjens, F.J., and Krevelen, D.W. van, Fuel, 33, pp. 88-103, (1954).

7. Seyler, C.A., J. Inst. Fuel, 16, pp. 134-141, (1943).

8. Stach, E., Mackowsky, M., Teichnuller, M., Taylor, G.H., Chandra, D., and Teichmuller, R., Stach's Textbook of Coal

Petrology, Borntraeger, Stuttgart, (1975).

9. Stach, E., and Michels, H.C., Geol. Jahrb, 71, pp. 113-143, (1955).

10. Roberts, F., and Young, J.Z., Proc. Inst. Elec. Eng. (London), פ9, $\mathrm{pp} .717-760,(1952)$.

11. Bomberger, D.R., and Duel, M., Trans. Am. Inst. Min. Metall. Pet. Eng., 229, pp. 65-69, (1964).

12. Bayer, J.L., Venton, G.H., and Hasse1, R.E., ASTM STP, 430, pp. 118-128, (1968).

13. McCartney, J.T., 0'Donne11, H.J., and Ergun, S., Fue1, 50, pp. 226-235, (1971).

14. Kojina, Sakurai, Suzai, and Hotogi, "An Automation of Coal Petrographical Analysis," Coal Sci. Congr., 11th, Kokkaido.

15. Davis, A., and Vastola, F.J., J. Microsc., 109, pp. 3-12, (1977).

16. Frompovicz, Zigmund J., "The Development and Application of an Automated Reflectance Microscopc for the Petrographic Analysis of Coal," a Thes is in Fucl Science, The Department of Materials Science and Engineering. Fuel Science Section, The Pennsylvania State University, 1978. 
17. Harlmann, Bruce M., "A Practical Method for the Determination of Inorganic Sulfur Concentration in Coal with an Abbreviated View of Pyrite Chord Length Distributions and a Maceral Type Analysis Resulting from Data Taken by an Automated Reflectance Microscope," a Paper in Fuel Science, The Department of Materials Science and Engineering, Fuel Science Section, The Pennsylvania State University, 1976.

18. Hoover, D., Kuehn, K.W., Davis, A., and Vastola, F., Geol. Soc. Am. Abst. Programs, $\underline{8}$, p. 925, (1976).

19. Dinger, Dennis, "New Strereological Method for Obtaining 3Dimensional Grain Size Distributions and Shape Factors from Planar Sections: Applications to Characterization of Mineral Matter in Coal," Ph.D. Thesis in Solid State Science, The Pennsylvania State University, 1975.

20. TIME, 113, No. 8, p. 51, (1979). 
APPENDIX A: Description of the Data-Acquisition System

Figure 17 is a schematic diagram of the data-acquisition system. The 6502 KIM-1 configuration is purchased already assembled on a printed circuit board. Interfacing to the processor is done via the address and data buses which are available thru an edge connector provided on the KIM-1 board. Although used as a dedicated machine for automated reficctance microscopy, the design of the dataacquisition system is quite general and by no means limited to this application. 


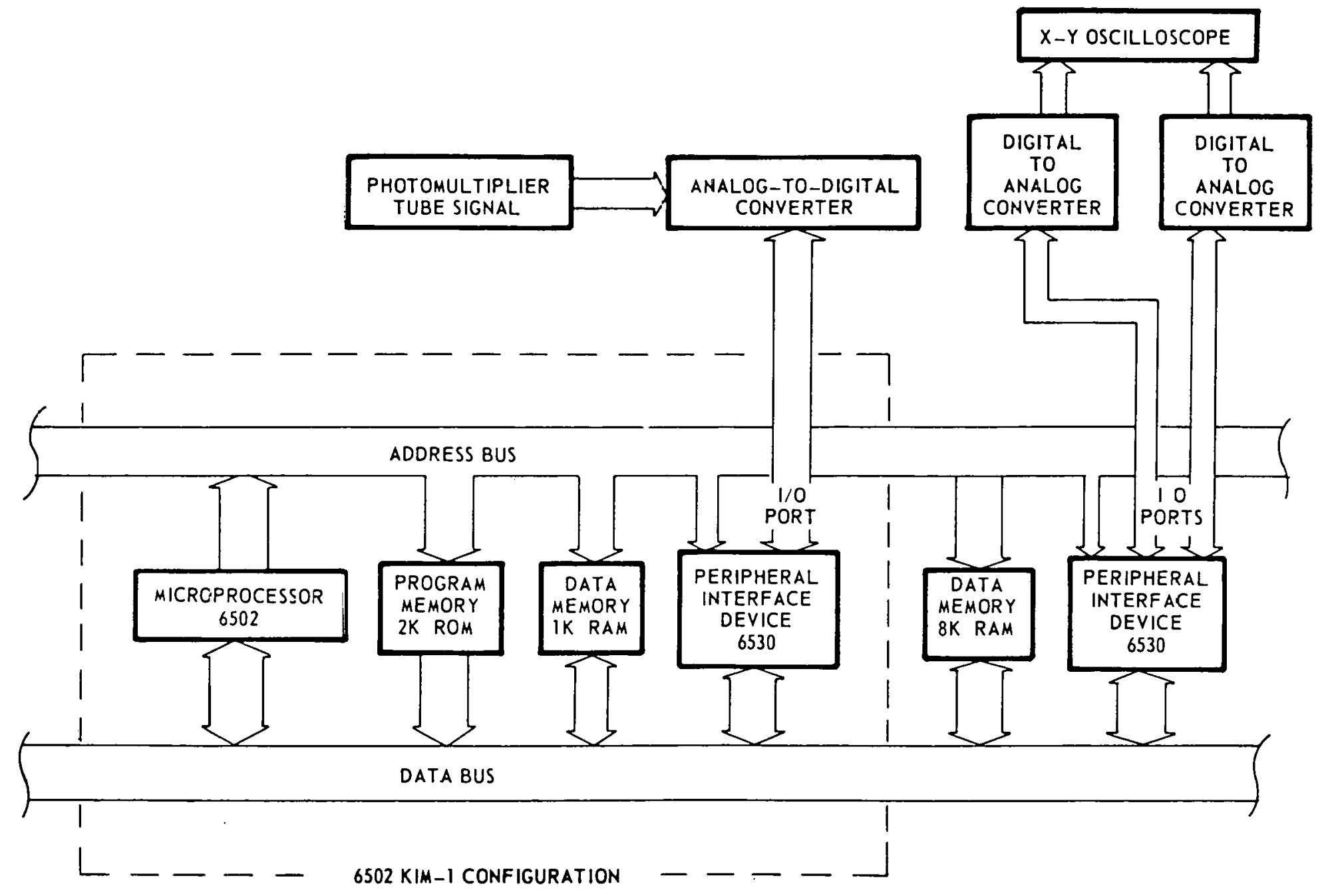

Appendix A: ORGANIZATION OF THE DATA-ACQUISITION UNIT BUILT FOR THE AUTOMATED REFLECTANCE MICROSCOPE SYSTEM 
APPENDIX B: Determination of a Particle Size Distribution from a Curve Representing a Collection of Spheres of Two Different Diameters.

Assume a collection of two populations of spheres of different diameters. A plot of the number of readings of a chord length versus chord length for independent collections of spheres would look like:
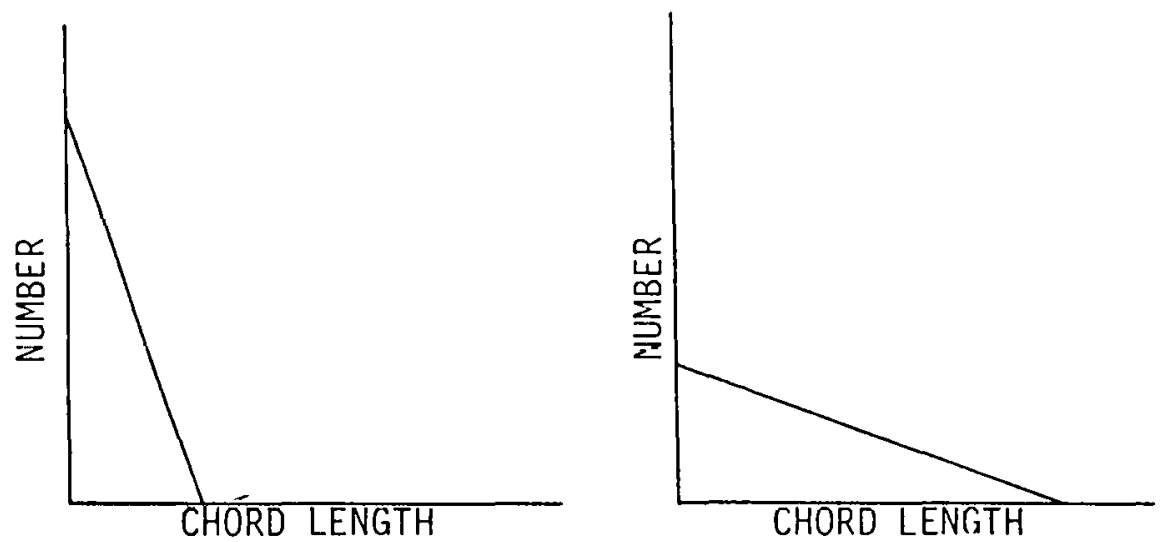

Now if these two sized spheres were mixed, a curve such as the following would be obtained:

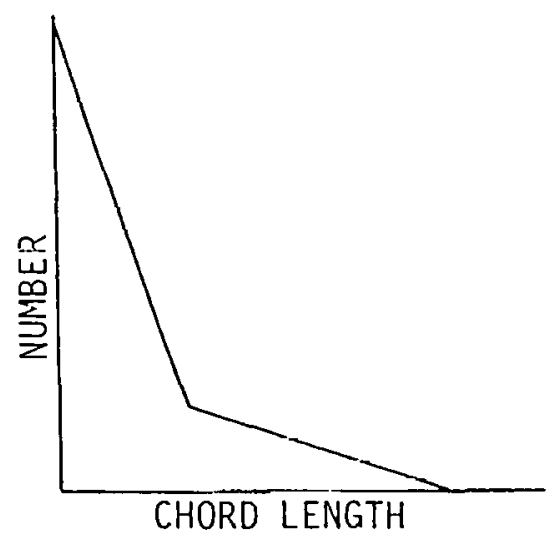

By regressing straight lines on the curve, a particle size distribution can be obtained. The slope of the calculated line indicates the diameter of the contributing spheres and the normalized area under the line the percentage that particular size contributes. Thus by an iterative procedure the original diameters of the spheres composing the curve and their proportions can be calculated. Detailed deconvolution methods can be found in Dinger (19). 
APPENUIX C: Description of the Connectivity Algorithm

The connectivity algorithm is based on the measurement of chord length. Chord length is determined by comparing successive reflectance readings. As long as each successive reading falls within a specified percent reflectivity $(0.09 \%$ in this study) of the previous reading, the length of the chord is extended. When the difference between any two successive readings falls out of the specified range, the number of readings that represent the measured chord length are processed. The reflectogram tables that represent each chord length less than or equal to the measured chord length are updated. In this way a series of reflectograms each representing chord lengths less than or equal to any given chord length can be obtained. Figure 18 shows the flow of the connectivity logic. 


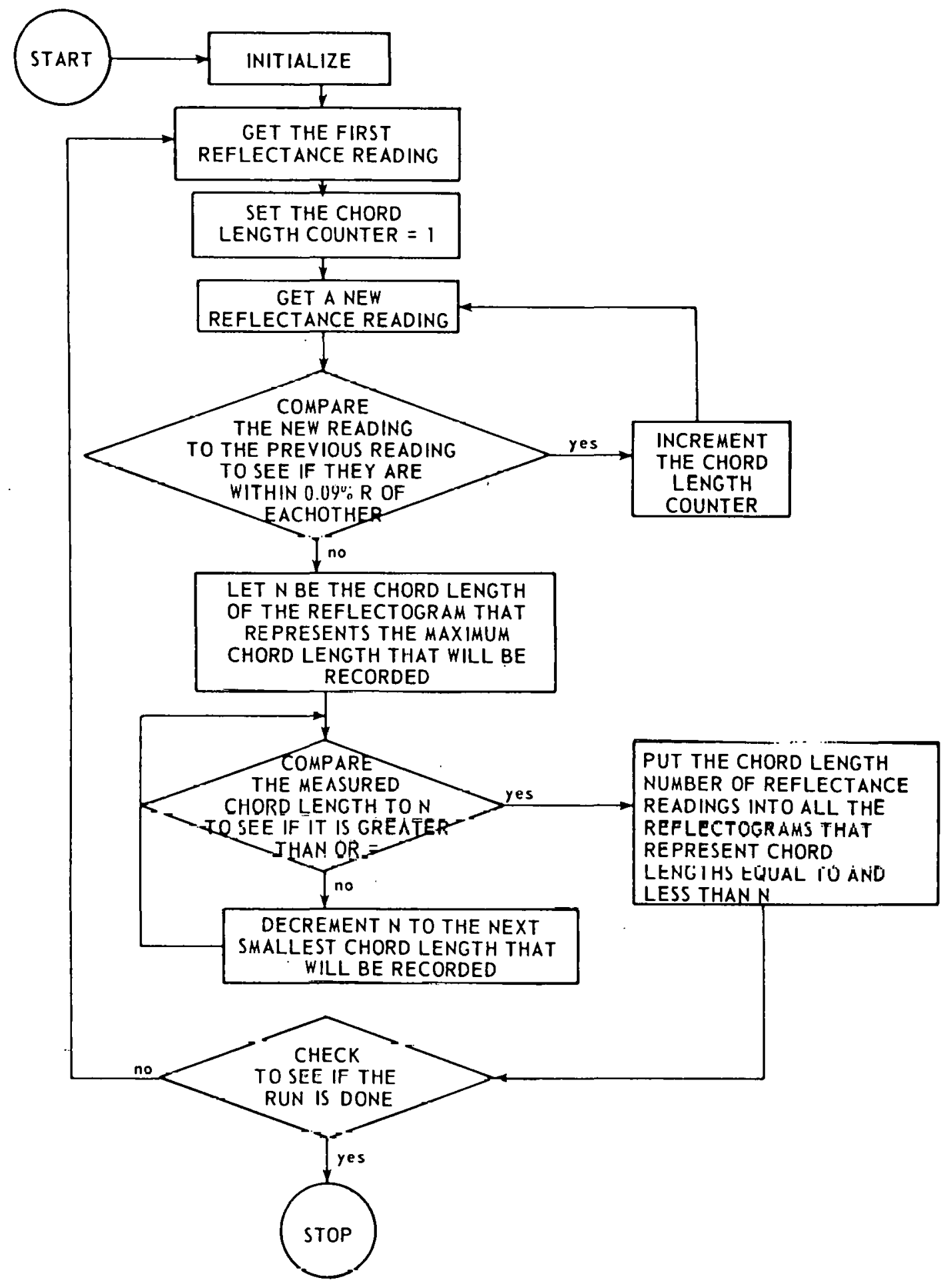

Appendix C: FLOW CHART OF THE CONNECTIVITY ALGORITHM 